\title{
Dust and nebular emission
}

\section{Models for normal galaxies}

\author{
P. Panuzzo ${ }^{1}$, A. Bressan ${ }^{2,1}$, G. L. Granato ${ }^{2}$, L. Silva ${ }^{3}$, and L. Danese ${ }^{1}$ \\ 1 Scuola Internazionale Superiore di Studi Avanzati, via Beirut 4, 34014 Trieste, Italy \\ 2 INAF, Osservatorio Astronomico di Padova, Vicolo dell'Osservatorio 5, 35122 Padova, Italy \\ 3 INAF, Osservatorio Astronomico di Trieste, via G. B. Tiepolo 11, 34131 Trieste, Italy
}

Received 14 February 2003 / Accepted 7 July 2003

\begin{abstract}
We present a model for nebular emission in star forming galaxies, which takes into account the effects of dust reprocessing. The nebular emissions (continuum emission, $54 \mathrm{H}$ and He recombination lines and 60 nebular lines from UV to IR) have been computed with CLOUDY and then included into GRASIL, our spectrophotometric code specifically developed for dusty galaxies. The interface between nebular emission and population synthesis is based on a set of pre-computed $\mathrm{H}$ II region emission models covering a wide range of physical quantities (metallicity, density, geometry and number of $\mathrm{HI}$, He I and O II ionizing photons). These quantities are fully adequate to describe the emission properties of the majority of starforming and starburst galaxies. Concerning the extinction properties of normal star forming galaxies, we are able to interpret the observed lack of correlation between the attenuation measured at $\mathrm{H} \alpha$ and in the UV band as a consequence of age selective extinction. We also find that, for these galaxies with modest SFR, the ratio FIR/UV provides the best constraints on the UV attenuation. The accurate treatment of lines and continuum in dusty galaxies also allows to deal with different SFR estimators in a consistent way, from the UV to radio wavelengths, and to discuss the uncertainties arising from the different physical conditions encountered in star forming galaxies. We provide our best estimates of SFR/luminosity calibrations, together with their expected range of variation. It results that SFR derived through $\mathrm{H} \alpha$, even when corrected for extinction using the Balmer decrement, is affected by important uncertainties due to age selective extinction. Another remarkable result is that SFR from UV luminosity corrected by means of the ratio FIR/UV has a small uncertainty. Finally, our model provides a calibration of SFR from radio luminosity; its value differs from estimates from other works, but we are able to reproduce the observed FIR/radio ratio. These results are relevant to estimates of the contribution of disk galaxies to the cosmic SFR at $z \leq 1$.
\end{abstract}

Key words. ISM: HII regions - galaxies: ISM - stars: formation - galaxies: evolution

\section{Introduction}

Massive stars in young stellar generations leave their signature as UV, ionizing photons and supernova ( $\mathrm{SN}$ ) explosions. Thus, ongoing star formation in galaxies is traced by the reprocessing of stellar radiation (line and continuum emission from the surrounding ionized gas, thermal emission by dust grains) and by synchrotron emission of SN accelerated electrons. The hydrogen recombination lines have been widely used as tracers of the current star formation rate (SFR), because of the direct proportionality between their intensity and the number of living massive stars. In the absence of dust, nebular emission models predict an almost constant ratio of $\mathrm{H} \alpha$ to $\mathrm{H} \beta$ for a large variety of environments (e.g. Osterbrock 1989); so, the dust absorption can be estimated by comparing the observed ratio to the theoretical one (Balmer decrement method).

Send offprint requests to: P. Panuzzo, e-mail: panuzzo@sissa.it
However, the discovery by IRAS of powerful and highly dust enshrouded starbursts (Soifer et al. 1986), namely the Luminous and Ultra Luminous Infrared Galaxies, has underlined the necessity to model nebular emission by consistently taking into account dust reprocessing. Moreover, an impressive wealth of information is coming from infrared spectral region. Both star formation and a possible nuclear activity contribute to infrared luminosity, but with different emission properties (Rigopoulou et al. 1999). The combination of different indicators (such as optical, near- and mid-infrared emission lines, mid-infrared PAH bands, shape of the continuum from the farultraviolet to the radio wavelengths) should then allow to obtain a fair picture of the star formation process and possible AGN contribution for a wide class of active galaxies.

Two models have been recently published in which spectral synthesis and photo-ionization codes are coupled. Charlot \& Longhetti (2001, henceforth CL01) combined a population synthesis code with the photo-ionization code CLOUDY (Ferland 2001), including an approximate prescription to 
estimate the dust absorption. According to CL01, the presence of dust increases the uncertainty of standard SFR estimators such as $\mathrm{H} \alpha$ or [O II] luminosities, from a factor of a few to several decades. Only the simultaneous consideration of several other lines and spectral features can reduce the uncertainty within a factor of a few. In a similar way, Moy et al. (2001, hereafter MRF01) interfaced the evolutionary synthesis model PÉGASE (Fioc \& Rocca-Volmerange 1997) with CLOUDY. In these models the dust processes are partially treated (extinction but not emission) using a simple screen approximation ${ }^{1}$.

However, growing evidence has been collected in the recent past showing that a physical understanding of dust effects in galaxies requires the inclusion of different environments, arranged in a rather complex geometry. A sophisticated treatment tends to become more and more important as the obscuration gets higher.

In this paper, we propose a new method to compute nebular emission in star forming galaxies. The method was implemented in the spectrophotometric synthesis model GRASIL ${ }^{2}$ (Silva et al. 1998), and then it was used to study the properties of normal star-forming galaxies.

The main advantage with respect to previous treatments is that GRASIL already provides a sound treatment of all the aspects of dust reprocessing, since it makes use of a geometry which is much closer to reality than a screen between the stars and the observer. Dust modeling is thus related to real physical parameters, describing the different distributions of dust and stars in molecular complexes and diffuse components, and their age dependence.

One of its important features is that it takes into account that, since stars are born in dense environments (the molecular clouds) and progressively become less obscured, the relative geometrical arrangement of dust and stars depends on the age of the stellar generation considered. Granato et al. (2000) have shown that this age-dependent extinction can explain the differences between the observed attenuation laws in normal and starburst galaxies (see also Poggianti et al. 2001).

GRASIL has been shown to reproduce the UV to radio continuum SEDs of galaxies, at low and high redshift, and in different evolutionary stages (Silva et al. 1998; Franceschini et al. 1998; Granato et al. 2000; Rodighiero et al. 2000; Granato et al. 2001).

The paper is organized as follows. In Sect. 2 we describe how we modeled stellar radiation and the computation of nebular emission from the single $\mathrm{H}$ II regions. Section 3 explains the calculation of nebular emission from galaxies and in the following sections our model is applied to the case of normal star-forming galaxies. In particular in Sect. 4 the different methods to estimate the attenuation are discussed. In Sect. 5 different SFR estimators, together with their uncertainties, are presented. In Sect. 6 we discuss the use of IR nebular lines. Thus, in Sect. 7 the main results of the paper are discussed,

\footnotetext{
1 Additional details on these models are given and compared to our method in Sect. 3.

2 The GRASIL code and updated information on it can be found at the URL:

http://web.pd.astro.it/granato/grasil/grasil.html.
}

and then summarized in the last section. Finally, some technical details of Sects. 2.2.1 and 3 are described in Appendices A and $\mathrm{B}$.

\section{Population synthesis with gas and dust}

In this section we summarize the main features of our population synthesis code GRASIL. More details can be found in Silva et al. (1998) and Granato et al. (2000). GRASIL represents galaxies by means of two main components characterized by different geometries: a spheroidal component (the bulge) and a disky component. Dust, that may be present in both components, is divided in two phases: i) dense molecular clouds (MCs), where star formation is active, and ii) diffuse medium (or cirrus).

Young stars are assumed to be born into MCs, and to leave them progressively as their age increases. As a consequence, the fraction of light of young simple stellar populations (SSPs) radiated inside MCs is a decreasing function of SSPs age, parameterized by the "escape time". Thus, the light of young stars will be attenuated by both the MCs and the cirrus, while older populations will only be affected by dust in the cirrus component. The molecular cloud is modeled as a thick spherical shell of dense gas (and dust) around a central point source, representing all the stellar content of the cloud. The time dependence of the escape fraction gives rise to the age-selective extinction because younger stellar generations are more attenuated than older ones.

Massive stars are supposed to ionize the surrounding medium and to give rise to the corresponding line and continuum nebular emissions. This radiation comes generally from within MCs and it is accordingly extinguished. However, it is worth noticing that, when the escape time is short enough, a significant number of ionizing photons can arise from star generations outside MCs and, consequently, we will also consider H II regions extinguished only by the cirrus component. To compute nebular emission we have used the photoionization code CLOUDY (version 94), as detailed in the following sections.

Since our interest is focused on the star formation process and the obscuration of star-forming regions, we do not include in our model the emission due to an Active Galactic Nucleus (AGN). We neglect the ionization due to UV radiation from post-AGB stars, since in presence of even a modest star formation activity their contribution is very low (Binette et al. 1994). Moreover, we neglect the contribution of shocks produced by SN explosions, that is typically low (Kewley et al. 2001). Finally, we do not include line emission from photo-dissociation regions and diffuse warm neutral/low ionized medium, although some fine structure IR lines are efficiently produced in these media. As a consequence their luminosities in our model will be only lower limits; this problem will be discussed in Sect. 2.3.

The final output of our model is a complete and detailed spectrum of star-forming galaxies, from far-UV to the radio wavelengths (an extension to X-ray band is in progress, Silva et al., in preparation), including stellar absorption features, nebular emission, dust and PAH emission. 


\subsection{Stellar radiation}

SEDs of stellar generations (SSP, simple stellar populations) have been computed by following the prescriptions outlined in Bressan et al. (1994) and in Silva et al. (1998). These SEDs cover a wide range in age and metal content. They can be computed for an arbitrary initial mass function (IMF) and allow the use of different atmosphere models, from the low-resolution (but covering a wide parameter space) Kurucz-Lejeune models (Kurucz 1993; Lejeune et al. 1998), to the intermediateresolution models of Pickles (1998) and Jacoby et al. (1984). In the latter two cases, which are derived from observed stars, the corresponding fluxes have been extended into the unobserved region by means of the Lejeune et al. (1998) models. The use of higher resolution models is particularly useful when dealing with emission lines superimposed to absorption features of the intermediate age populations. As for the most massive stars, we have adopted the atmospheric models by Schaerer et al. (1996) for mass-losing blue supergiants, and the models by Schmutz et al. (1992) in the Wolf Rayet (WR) phase. It will be stressed below that the emission from $\mathrm{H}$ II regions has been computed in a way that is almost independent of the detailed shape of the adopted SEDs of stellar populations.

\subsection{H II regions model}

The emission spectrum from a single H II region depends on two main ingredients: the SED of the ionizing star cluster and the properties of the excited gas. For a given stellar evolution scenario, the SED is determined by the IMF and the total mass, age and metallicity of the cluster, as well as by the adopted model atmospheres. On the other hand, the important properties of the excited gas are the density, the chemical composition and the geometry. In a galaxy the situation is more complicated and star forming regions possibly have different ages and different metallicities. In starbursts, star clusters of different ages and metallicities may coexist within the same star forming region. To compute the line emission intensities, one should consider the spectrum of the ionizing source, provided by the recent star formation history, and use a photoionization code with suitable values of the gas parameters. To reduce the computing cost, one can use a pre-built library of line intensities, corresponding to different ages of the ionizing SSP and different metal contents of both the SSP and the gas. This still requires different libraries for different masses of the ionizing SSPs, assumed IMFs and model atmospheres. It becomes particularly time consuming in applications requiring a large number of models.

Our approach has been to pick out the physical parameters which actually affect the emission properties of $\mathrm{H}$ II regions. The analysis described in the following section shows that the emission line spectrum of an $\mathrm{H}$ II region with fixed gas properties (metallicity, density and geometry) is described with reasonable precision by only three quantities: the number of ionizing photons for $\mathrm{HI}$, He I and O II $\left(Q_{\mathrm{H}}, Q_{\mathrm{He}}\right.$, and $Q_{\mathrm{O}}$, defined in Eq. (2)). This method allows us to get rid of the particular SSP model and IMF. In fact, different ionizing sources that provide the same values of $Q_{\mathrm{H}}, Q_{\mathrm{He}}$, and $Q_{\mathrm{O}}$, will produce the same emission line spectra, within a reasonable accuracy.
Thus, we computed a library of photoionization models as a function of $Q_{\mathrm{H}}, Q_{\mathrm{He}}$, and $Q_{\mathrm{O}}$. When estimating the actual line emission due to a given stellar population, we compute $Q_{\mathrm{H}}$, $Q_{\mathrm{He}}$, and $Q_{\mathrm{O}}$ from the corresponding SED, and then interpolate the value from the above library.

The H II region library can be freely retrieved through the GRASIL web page (see Sect. 1).

In the following, we will describe the adopted procedure and test its accuracy.

\subsection{1. lonizing spectra}

In order to have photoionization models as a function of $Q_{\mathrm{H}}$, $Q_{\mathrm{He}}$, and $Q_{\mathrm{O}}$, we approximate real SEDs with piece-wise blackbodies, in a way such that the space of $Q$ parameters is covered as much as possible.

The Lyman continuum spectra of typical young stellar populations show two prominent discontinuities, at $504.1 \AA$ and $227.8 \AA$, corresponding respectively to the ionizing energies for He I and He II. At wavelengths smaller than $227.8 \AA$ there are very few photons. Between the Lyman break, He I break, and He II break, spectra can be reasonably well represented by blackbodies. Thus, when computing the libraries, we used the following functional representation of the ionizing SEDs:

$$
F_{v}=\left\{\begin{array}{ll}
0 & \text { for } \lambda<227.8 \AA \\
A_{\mathrm{He}} B_{v}\left(T_{\mathrm{He}}\right) & \text { for } 227.8 \AA<\lambda<504.1 \AA \\
A_{\mathrm{H}} B_{v}\left(T_{\mathrm{H}}\right) & \text { for } 504.1 \AA<\lambda<911.76 \AA \\
A_{\mathrm{ni}} B_{v}\left(T_{\mathrm{ni}}\right) & \text { for } 911.76 \AA<\lambda
\end{array},\right.
$$

where $B_{v}(T)$ is the Planck function at temperature $T$. This function depends on the 6 quantities $A_{\mathrm{He}}, T_{\mathrm{He}}, A_{\mathrm{H}}, T_{\mathrm{H}}, A_{\mathrm{ni}}$ and $T_{\mathrm{ni}}$. However, we found that the SEDs of young stellar populations can be well approximated by writing these 6 quantities in terms of only three parameters. These are the numbers of ionizing photons for $\mathrm{HI}, \mathrm{He}$ I and O II $\left(Q_{\mathrm{H}}, Q_{\mathrm{He}}\right.$, and $\left.Q_{\mathrm{O}}\right)$ :

$$
Q_{\mathrm{H}}=\int_{v_{\mathrm{H}}}^{\infty} \frac{F_{v}}{h v} \mathrm{~d} v, Q_{\mathrm{He}}=\int_{v_{\mathrm{He}}}^{\infty} \frac{F_{v}}{h v} \mathrm{~d} v, Q_{\mathrm{O}}=\int_{v_{\mathrm{O}}}^{\infty} \frac{F_{v}}{h v} \mathrm{~d} v,
$$

where $v_{\mathrm{H}}, v_{\mathrm{He}}$ and $v_{\mathrm{O}}$ are the photoionization threshold frequencies for respectively $\mathrm{HI}$, He I, and O II (the last one correspond to $\lambda_{\mathrm{O}}=350.7 \AA$ ). The relationship between the $Q$ values and the parameters of analytical spectra is described in Appendix A.

We built a library for a grid of values of $Q_{\mathrm{H}}, Q_{\mathrm{He}}$, and $Q_{\mathrm{O}}$, and for different assumptions on the gas density, metallicity and filling factor $(\epsilon)$. When the filling factor is different from 1 , the gas in the H II region is supposed to be divided in small clumps, and $\epsilon$ is defined as the ratio between the volume occupied by the clumps and the total volume of the H II region. The gas density refers to the hydrogen density inside the clumps. H II regions are assumed to be spherical and ionization bounded, with a constant density along the radius, and with a covering factor of 1 . The abundance of elements respect to $\mathrm{H}$ are from McGaugh (1991). These abundances are relative to gas phase, thus they already account for the fraction stored in dust (depletion). 
The characteristics of dust inside $\mathrm{H}$ II regions and its effects on the emerging emission are still poorly known. However, it is reasonable that the main effect is that a fraction $f_{\mathrm{d}}$ (typically $\sim 30 \%$, see DeGioia-Eastwood 1992) of ionizing photons is absorbed by dust. To approximate the consequence, one can multiply the nebular emission of a dust-free model by a factor $1-f_{\mathrm{d}}$. Thus, absorption by internal dust has not been considered in $\mathrm{H}$ II region models presented here. Moreover, including dust inside photo-ionization models would introduce new free parameters, and the library could be coherently used only in some specific cases.

It is worth noticing that the emission properties of the ionized gas (i.e. the ratio of different line intensities) at a fixed density do not depend directly on the geometry of the gas, while it shows a dependence on the ionization parameter $U$, that expresses the number of ionizing photons per particle. For a thin spherical shell at distance $R$ from a central ionizing source, the ionization parameter is $U=Q_{\mathrm{H}} /\left(4 \pi R^{2} c n_{\mathrm{H}}\right)$; it can be shown (see Appendix B) that the volume averaged ionization parameter $\langle U\rangle$ for a sphere, with density $n_{\mathrm{H}}$ and filling factor $\epsilon$, is

$\langle U\rangle=\frac{3 \alpha_{\mathrm{B}}^{2 / 3}(\mathrm{H}, T)}{4 c}\left(\frac{3 Q_{\mathrm{H}} n_{\mathrm{H}} \epsilon^{2}}{4 \pi}\right)^{1 / 3}$,

where $\alpha_{\mathrm{B}}(\mathrm{H}, T)$ is the recombination coefficient of hydrogen in the case $\mathrm{B}$. Thus, in our geometry $\langle U\rangle$ is proportional to $\left(Q_{\mathrm{H}} n_{\mathrm{H}} \epsilon^{2}\right)^{1 / 3}$ while for the plane parallel geometry $U \propto Q_{\mathrm{H}} / n_{\mathrm{H}}$.

As a consequence, our results remain also valid for different geometries and/or filling factors, provided that we renormalize $Q_{\mathrm{H}}$ to maintain the same value of $\langle U\rangle$.

The computed lines are $54 \mathrm{H}$ and $\mathrm{He}$ I recombination lines and 60 nebular line of other elements with wavelength from UV to FIR. The complete list of lines and the space of parameters covered by the library can be found in the web page.

\subsubsection{Nebular continuum emission}

The nebular continuum emission can be easily evaluated using the Strömgren theory for the case B approximation (see Osterbrock 1989) and added to the emerging spectrum.

We get

$E_{v}=Q_{\mathrm{H}} \frac{\gamma_{v}(\mathrm{H}, T)}{\alpha_{\mathrm{B}}(\mathrm{H}, T)}+Q_{\mathrm{He}} \frac{\gamma_{v}(\mathrm{He}, T)}{\alpha_{\mathrm{B}}(\mathrm{He}, T)}$,

where $\gamma_{v}(\mathrm{He}, T)$ and $\gamma_{v}(\mathrm{H}, T)$ are the total emission coefficients and $\alpha_{\mathrm{B}}(\mathrm{He}, T)$ and $\alpha_{\mathrm{B}}(\mathrm{H}, T)$ are the recombination coefficients, respectively of helium and hydrogen. In the former coefficients we take into account recombination, free-free emission of H II and He II and the Ly $\alpha$ two photons decay emission. Free-free emission is computed as described in Bressan et al. (2002), while other coefficients are from Burgess \& Summers (1976), Aller (1984), Osterbrock (1989), Ferland (1980) and Nussbaumer \& Schmutz (1984). The He III contribution to the nebular emission is very small and can be neglected.

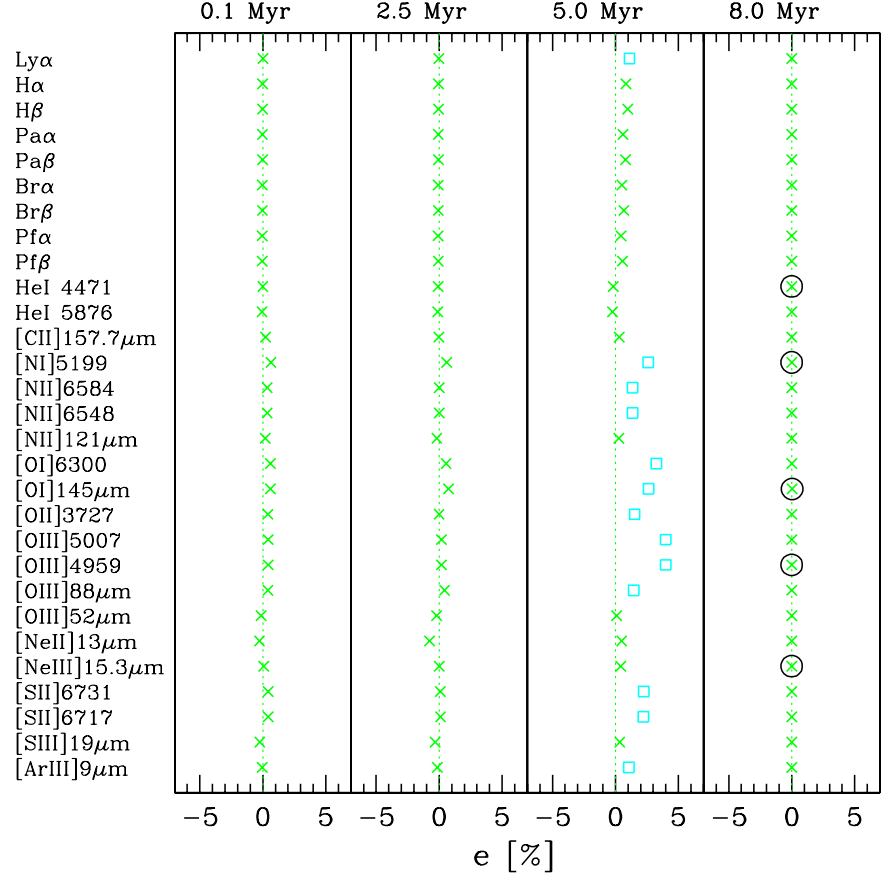

Fig. 1. Differences between H II region models excited by SSP spectra with and without high energy photons. Crosses are for difference smaller than $1 \%$, empty squares for difference between $1 \%$ and $5 \%$; circled symbols refer to very low luminosity lines. Gas and star metallicity is 0.008 ; the stellar mass is $3 \times 10^{4} M_{\odot}$.

\subsubsection{Accuracy}

Before proceeding further, we need to analyze the errors introduced by adopting the analytic SEDs (Eq. (1)) instead of the real SSP with the same $Q$ values.

A first problem is that our analytical spectra neglect photons with energy higher than the He II ionization threshold (hereafter we will refer to these photons as high energy photons). This does not allow a realistic evaluation of the luminosity of the He II lines. However, nebular He II lines are superimposed to the generally more intense photospheric lines, emitted in the stellar atmospheres of WR stars (Conti 1991; Schaerer et al. 1999). We also cannot compute line intensities of highly ionized elements, such as O IV or Ne IV. These lines are typically either not observed, or very faint in pure star forming galaxies, so that they are often interpreted as a signature of AGN activity. The impact of neglecting high energy photons on other lines is quantified by comparing the calculated emission of $\mathrm{H}$ II regions excited by SSP spectra with and without high energy photons, normalized in order to have the same $Q_{\mathrm{H}}$. The results (for some ages and metallicities) are shown in Fig. 1 for a Salpeter IMF between 0.15 and $120 M_{\odot}$. The difference in the two cases is always $<10 \%$, and typically $<1 \%$; the highest difference appears at SSP ages at which the WR stars produce their maximum flux in high energy photons. We conclude that the lack of high energy photons is not very important, at least for the lines considered here.

The comparison between line intensities of $\mathrm{H}$ II region models excited by an SSP spectrum and those excited by a parametric spectrum with the same values of $Q_{\mathrm{H}}, Q_{\mathrm{He}}$, and $Q_{\mathrm{O}}$ 


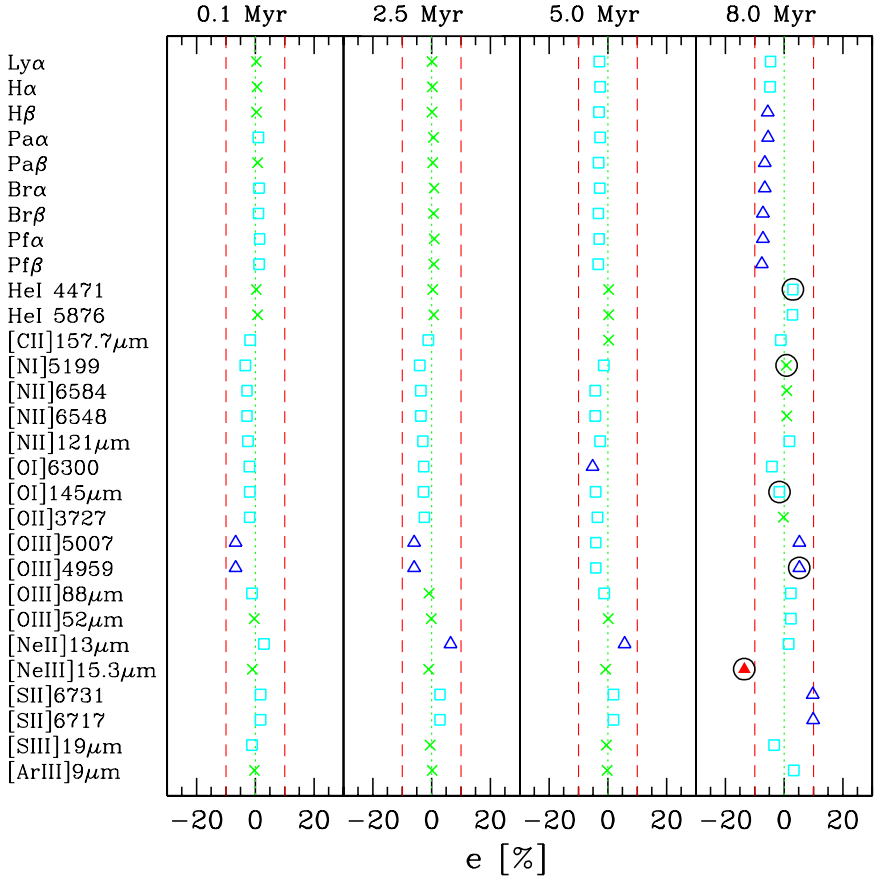

Fig. 2. Differences between H II region models excited by SSP spectra and parametric spectra with the same $Q$ s. Empty triangles represent differences between $5 \%$ and $10 \%$, filled triangles represent differences greater than $10 \%$.

is shown in Fig. 2. Typical differences in the emission lines are lower than $10 \%$, with the larger ones arising at lower values of $Q_{\mathrm{H}}$, i.e. older ages. Most of them are due to discrepancies between the SSP and parametric SEDs at wavelengths longer than the Lyman break. Indeed, photons with wavelengths corresponding to Lyman serie lines can be efficiently absorbed and re-emitted in optical lines. When the ionizing flux decreases with respect to the non-ionizing one, this effect can be important. Notice however that, when computing the lines emitted by a combination of $\mathrm{H}$ II regions excited by clusters with different ages, the most important contribution arises from the youngest populations.

As noticed in previous works (see e.g. Stasińska et al. 2001 or Rubin et al. 2001), the photo-ionization models cannot be accurate when the gas metallicity exceeds the solar value, for several reasons. As the metallicity increases, fine structure infrared lines of metals (that depend little on temperature) dominate the cooling processes, but their transition probabilities have not yet been well determined. On the other hand, optical collisionally excited lines, that depend strongly on the electronic temperature, become uncertain because the temperature is regulated by infrared lines. Furthermore, the thermal instability of the gas affects the line intensity when the temperature is low and the metallicity is high. Finally, a further source of uncertainty at high metallicity is due to the poorly known depletion of metals into dust grains. In order to minimize this problem, we used a set of abundances measured by McGaugh (1991) in the gas phase.

In order to check the flexibility of our procedure, we compare in Fig. 3 the results obtained by repeating the above test when adopting a very different IMF, namely a top-heavy

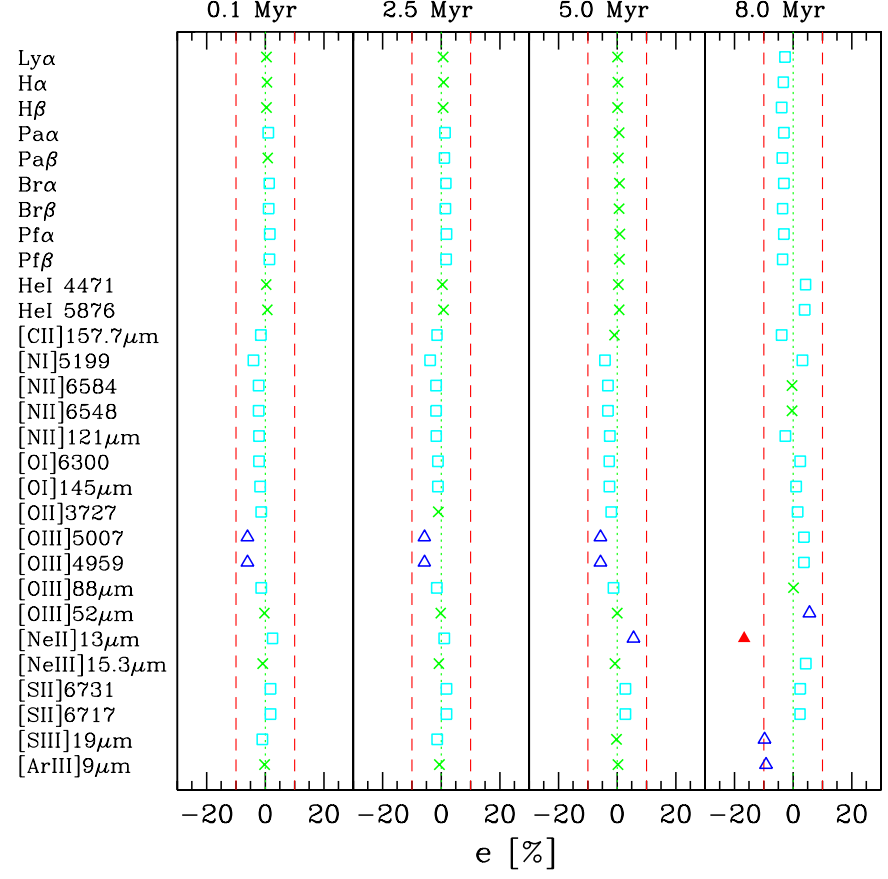

Fig. 3. As Fig. 2, but with SSPs with a top-heavy modified Kennicutt's IMF.

modified Kennicutt's IMF $(\mathrm{d} \log N / \mathrm{d} \log m=-0.4$ for $0.15 M_{\odot}<m<1 M_{\odot}$ and $\mathrm{d} \log N / \mathrm{d} \log m=-1$ for $1 M_{\odot}<$ $m<120 M_{\odot}$, Kennicutt 1983). The differences between H II region models excited by SSP spectra and analytical ones with the same $Q$ values are not larger than in the case of the Salpeter IMF, despite the very different slope of the IMF.

In conclusion, our analytical spectra can reproduce with a good accuracy the nebular emission properties of $\mathrm{H}$ II regions excited by star clusters with different IMF, age and metallicity by only making use of the three quantities $Q_{\mathrm{H}}, Q_{\mathrm{He}}$, and $Q_{\mathrm{O}}$. Obviously this accuracy could be improved by adding some other quantity to describe the SED with more details, but this would dramatically increase the number of $\mathrm{H}$ II region models used for the library.

\section{3. $C$ and $O$ infrared lines}

CI, C II and OI fine structure infrared lines are produced not only in H II regions, but also in warm neutral and ionized interstellar medium and photodissociation regions (PDR). [O I] $63.2 \mu \mathrm{m}$ and $145.5 \mu \mathrm{m}$, [C I] $369 \mu \mathrm{m}$ and $610 \mu \mathrm{m}$ infrared lines have upper energy levels respectively of $228 \mathrm{~K}, 326 \mathrm{~K}$, $62.5 \mathrm{~K}$ and 23.6 K (Kaufman et al. 1999), so they can easily be produced in neutral medium. Because carbon has a ionization potential $(11.26 \mathrm{eV})$ which is lower than $\mathrm{H}$, the $\mathrm{C}$ II ion is present in PDR and in neutral medium illuminated by far-UV stellar radiation. Indeed, the [C II] $157.7 \mu \mathrm{m}$ line is the most important coolant of warm neutral medium. The relative contribution of different media to these lines is still a matter of debate (Heiles 1994; Malhotra et al. 2001). Since our model does not include PDR or neutral gas emission, the luminosities predicted for these lines must be taken as a lower limit. 


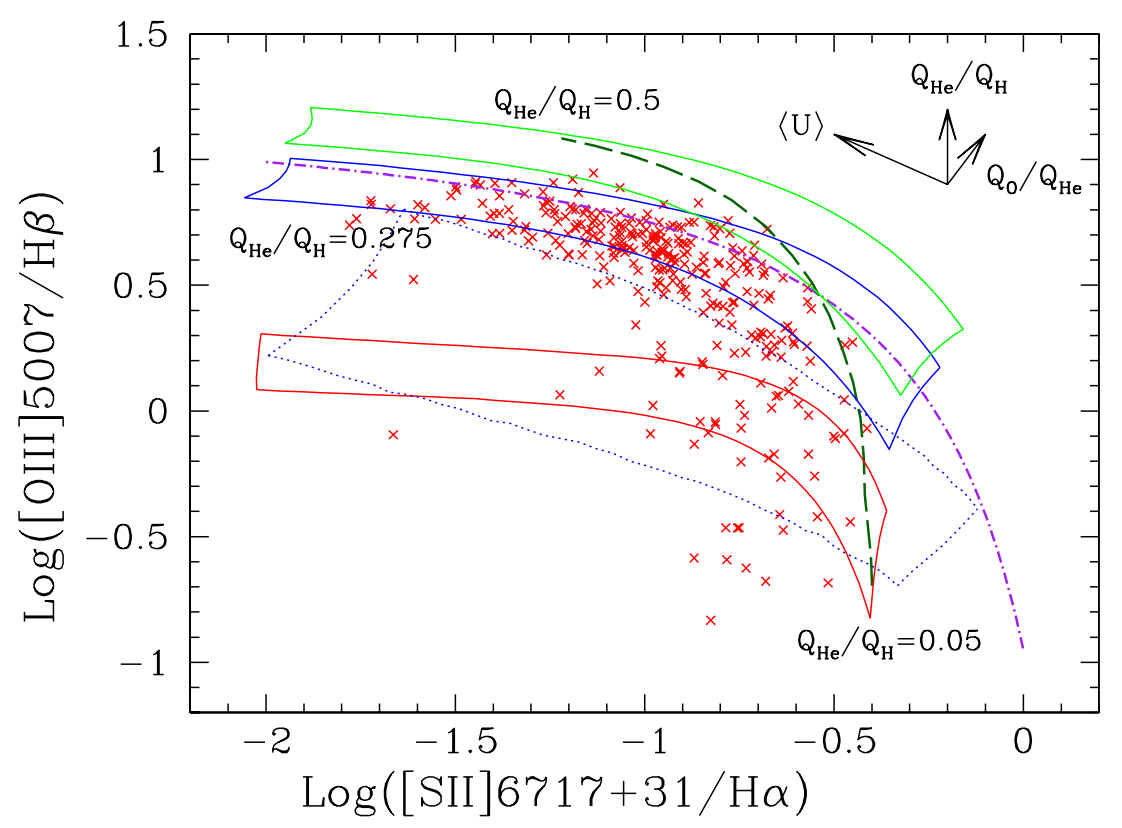

Fig. 4. Diagnostic diagram $\log ([\mathrm{O} \mathrm{III}] / \mathrm{H} \beta)$ vs. $\log ([\mathrm{S} \mathrm{II}] / \mathrm{H} \alpha)$. Crosses are observed H II galaxies from Dessauges-Zavadsky et al. (2000). The three solid closed lines show the regions occupied by $\mathrm{H}$ II region models from our library with three values of $Q_{\mathrm{He}} / Q_{\mathrm{H}}(0.5,0.275$ and 0.05$)$. The arrows show shifts at increasing $\langle U\rangle, Q_{\mathrm{He}} / Q_{\mathrm{H}}$, or $Q_{\mathrm{O}} / Q_{\mathrm{He}}$. $\log (\langle U\rangle)$ ranges from -0.867 to -3.534 , and $Q_{\mathrm{O}} / Q_{\mathrm{He}}$ from 0.13 to 0.63 . The models have a gas metallicity of 0.004 , while the closed dotted line shows the position of models with solar metallicity and $Q_{\mathrm{He}} / Q_{\mathrm{H}}=0.275$. All the models have a hydrogen density of $10 \mathrm{~cm}^{-3}$. The thick long dashed line separates the region occupied by $\mathrm{H}$ II galaxies (on the left) from the AGN region, as empirically defined by Veilleux \& Osterbrock (1987); the thick dot-dashed line delineates the same separation computed by Kewley et al. (2001).

\subsection{Comparison with observed $\mathrm{H}$ II galaxies}

In Fig. 4 we compare our HiI library with observations of a sample of H II galaxies (crosses) from Dessauges-Zavadsky et al. (2000), a revision of the Terlevich's catalogue (Terlevich et al. 1991). H II galaxies are typically defined as galaxies with spectra very similar to those of H II regions; these objects are interpreted as bursting dwarf galaxies. In the figure we show the dependence of models on $\langle U\rangle$ (or $Q_{\mathrm{H}}$ via the Eq. (3)) and on the hardness of the ionizing SED, expressed by $Q_{\mathrm{He}} / Q_{\mathrm{H}}$ and $Q_{\mathrm{O}} / Q_{\mathrm{He}}$.

The library covers the region of the $[\mathrm{O}$ III] $/ \mathrm{H} \beta$ vs. [S II]/H $\alpha$ diagnostic diagram populated by observed H II galaxies. Some models fall in the region of the diagnostic diagrams occupied by AGNs; these models are produced by the hardest spectra in the library $\left(Q_{\mathrm{He}} / Q_{\mathrm{H}} \simeq 0.5\right)$, harder than the spectra that can be built with true SSPs.

\section{Nebular emission from a star-forming galaxy}

When considering a star-forming galaxy as a whole, we need to integrate the emission lines resulting from the effects of different stellar populations. This cannot simply be obtained by calculating the ionizing photon fluxes $Q_{\mathrm{H}}, Q_{\mathrm{He}}$, and $Q_{\mathrm{O}}$ of the integrated spectrum and by getting the corresponding line intensities from our interpolation tables. Indeed, the intensity of metal lines also depends on the hardness of the ionizing spectra and on the ionization parameter. To obtain a more realistic description of the nebular emission, it is then necessary to model the formation and evolution of the population of H II regions, each one characterized by a different intensity, hardness and ionization parameter. This may be accomplished by splitting the recent SF history in subsequent episodes of suitable duration, and by computing their separate contributions to the emission lines.

The total emission in the line $l\left(E_{l}\right)$ at the epoch of observation $T$, may be written as

$E_{l}=\sum_{j} N_{\mathrm{HII}, j} E_{l}^{*}\left(Q_{\mathrm{H}, j}^{*}, Q_{\mathrm{He}, j}^{*}, Q_{\mathrm{O}, j}^{*}, Z_{\mathrm{gas}}\right)$,

where $N_{\mathrm{HII}, j}$ is the number of $\mathrm{H}$ II regions that have formed in the time interval $\left[T-t_{j+1}, T-t_{j}\right], Q_{\mathrm{H}, \mathrm{He}, \mathrm{O}, j}^{*}$ are the corresponding ionizing photon fluxes, $Z_{\mathrm{gas}}$ is the current metallicity of the gas and $E_{l}^{*}$ is the emission from the single $\mathrm{H}$ II region.

$N_{\mathrm{H} I \mathrm{I}, j}$ is obtained by assuming that each H II region is illuminated by a single cluster of total mass $M^{*}$, so that

$N_{\mathrm{H} \text { II }, j}=\frac{\int_{t_{j}}^{t_{j+1}} \Psi(T-t) \mathrm{d} t}{M^{*}}$,

where $\Psi$ is the SFR. $Q_{\mathrm{H}, j}^{*}$ for the single $\mathrm{H}$ II region at different ages can be obtained by

$$
\begin{aligned}
Q_{\mathrm{H}, j}^{*} & =\int_{v_{\mathrm{H}}}^{\infty} \frac{L_{v, j}^{*}}{h v} \mathrm{~d} v \\
& =\frac{\int_{v_{\mathrm{H}}}^{\infty} \int_{t_{j}}^{t_{j+1}} \Psi(T-t)\left(S_{v}(t, Z(T-t)) / h v\right) \mathrm{d} t \mathrm{~d} v}{N_{\mathrm{H} \mathrm{II}, j}}
\end{aligned}
$$

where we have emphasized the dependence on the metallicity of the ionizing spectra. Similar expressions hold for $Q_{\mathrm{He}, j}^{*}$, and $Q_{\mathrm{O}, j}^{*}$. 
The emission properties of the $j$ population depend on the ratios $Q_{\mathrm{He}, j}^{*} / Q_{\mathrm{H}, j}^{*}$ and $Q_{\mathrm{O}, j}^{*} / Q_{\mathrm{He}, j}^{*}$, that are constrained by the age of the $j$ population, and on the average ionization parameter of the $\mathrm{H}$ II regions $\langle U\rangle_{j}^{*}$.

The correspondence between the integrated Lyman continuum of the population $j$ (expressed by $N_{\mathrm{H} \mathrm{II}, j} \times Q_{\mathrm{H}, j}^{*}$ ) and $\langle U\rangle_{j}^{*}$ is determined by the stellar mass $M^{*}$ of ionizing clusters through Eqs. (6), (7) and

$\langle U\rangle_{j}^{*}=\frac{3 \alpha_{\mathrm{B}}^{2 / 3}}{4 c}\left(\frac{3 Q_{\mathrm{H}, j}^{*} n_{\mathrm{H}} \epsilon^{2}}{4 \pi}\right)^{1 / 3}$.

As shown in Fig. 4, H II galaxies exhibit a big variation of the ionization parameter value, which means that the value of stellar mass $M^{*}$ and/or of the filling factor are subject to substantial variations. Observations (e.g. Kennicutt 1984) suggest that $M^{*}$ varies approximately from 1000 to $10^{6} M_{\odot}$ and $\epsilon$ from 0.1 to 0.001 .

The duration of the subsequent episodes in which we split the SF history should be chosen by considering the evolution of the shape of the ionizing spectra. A lower limit is set by considering that each $\mathrm{H}$ II region will be illuminated by all the stars formed within the finite formation time of a typical star cluster, that we assume to be $1 \mathrm{Myr}$ (see e.g. Fuente et al. 2001).

As remarked in Sect. 2.2.1, the presence of dust inside $\mathrm{H}$ II regions can be important. Thus one can multiply the nebular emission obtained from Eq. (5) by a factor $1-f_{\mathrm{d}}$.

Finally, the emission lines are extinguished in the same way as the stellar populations that produced them (see Sect. 2).

In galaxies with normal dust content, almost all the photons in the resonant $\operatorname{Ly} \alpha$ line are reprocessed via two-photon decay and dust absorption and re-emission. In our model we assume that all Ly $\alpha$ photons decade via two-photons emission, and then these photons are possibly absorbed by dust. Anyhow, their contribution to the IR luminosity amounts to $\leq 10 \%$. Only in the case of very low dust content, the relative contribution of absorbed Ly $\alpha$ photons increases respect to the contribution from absorbed stellar continuum and may be significant. A detailed treatment of the Ly $\alpha$ transfer should also include the effects of winds and outflows, which strongly reduce the resonant scattering.

We conclude this section by comparing our method with other approaches found in literature.

Fioc \& Rocca-Volmerange (1997) computed $Q_{\mathrm{H}}$ from the integrated spectrum and then analytically obtain the $\mathrm{H} \beta$ line intensity. Other hydrogen and metals lines are then rescaled in fixed proportions. Moy et al. (2001) have improved the previous model by coupling the integrated spectrum with CLOUDY.

A more detailed analysis of emission properties in star forming galaxies is by CL01. These authors obtained the emission from the galaxy by summing the line intensities of separate SSPs of given constant average metallicity, weighted by the corresponding SFR. CL01 also have a more detailed model for dust absorption (see Charlot \& Fall 2000) that accounts for an age selective extinction.

Compared to MRF01, our model accounts for the coexistence of different populations of $\mathrm{H}$ II regions, while at variance with CL01, it quickly computes the line emissions for different choices of $M^{*}$ and gas density. Also, the $\mathrm{H}$ II regions library can be used for different IMFs, and we relax the hypothesis that the metallicities of the excited gas and of the ionizing stellar population are the same (but a non-negligible difference is expected only in extreme situations). Finally, our library can be easily used for other population synthesis models.

\section{Attenuation properties of normal star forming galaxies}

In star forming galaxies, the intrinsic UV luminosity is directly related to the star formation rate; on the other hand, the UV flux is heavily affected by extinction. Therefore it is important to understand how dust absorption affects the UV luminosity and, as a consequence, the SFR estimates.

In this section we discuss several methods to estimate the attenuation in normal star forming galaxies by considering the UV, optical emission lines and FIR properties. We will show that observations require the extinction of different stellar populations to vary with age, with younger populations suffering a larger extinction.

Notice that we will use the term attenuation when it refers to the amount of light lost at a give wavelength from a complex system (i.e. a galaxy) and the term extinction for the light lost from a simple source (as a star) with dust along the line of sight.

\subsection{Estimation of UV attenuation}

Attenuation in local star forming galaxies can be derived in several ways.

A common method is to relate the UV attenuation to the attenuation in $\mathrm{H} \alpha$. Calzetti (1997) suggested that the attenuation of the stellar continuum is only a fraction $(\sim 0.44)$ of the attenuation of the ionized gas. By extending this assumption to the UV while adopting a suitable extinction law, it is straightforward to obtain (at $\lambda_{\mathrm{UV}}=2000 \AA$, e.g. Buat et al. 2002)

$A_{2000}=1.6 A_{\mathrm{H} \alpha}$.

The gas attenuation at $\mathrm{H} \alpha$ is derived from the Balmer decrement:

$A_{\mathrm{H} \alpha}=1.086 \frac{1}{e_{\beta \alpha}-1} \ln \left(\frac{j_{\mathrm{H} \beta}}{j_{\mathrm{H} \alpha}} \frac{L_{\mathrm{H} \alpha}}{L_{\mathrm{H} \beta}}\right)$,

where $e_{\beta \alpha}=\tau_{\mathrm{H} \beta} / \tau_{\mathrm{H} \alpha}$ is derived from the adopted extinction law and $j_{\mathrm{H} \beta} / j_{\mathrm{H} \alpha}$ is the ratio of $\mathrm{H} \beta$ and $\mathrm{H} \alpha$ emission coefficients (typically assumed 2.87 at an electronic temperature of $10^{4} \mathrm{~K}$, see Osterbrock 1989).

An alternative method to derive the UV attenuation is to consider the reprocessing of star light into the infrared emission. Meurer et al. (1999) (see also Calzetti et al. 2000) assumed that the energy re-emitted by dust in a galaxy is provided primarily by the UV flux of young star populations. They related (Eq. (10) of their paper) the ratio between the FIR and UV fluxes (now $\lambda=1600 \AA$ ), $F_{\text {FIR }} / F_{1600}$, to the UV attenuation by:

$A_{1600}=2.5 \log \left(\frac{F_{\mathrm{FIR}}}{0.84 F_{1600}}+1\right)$ 


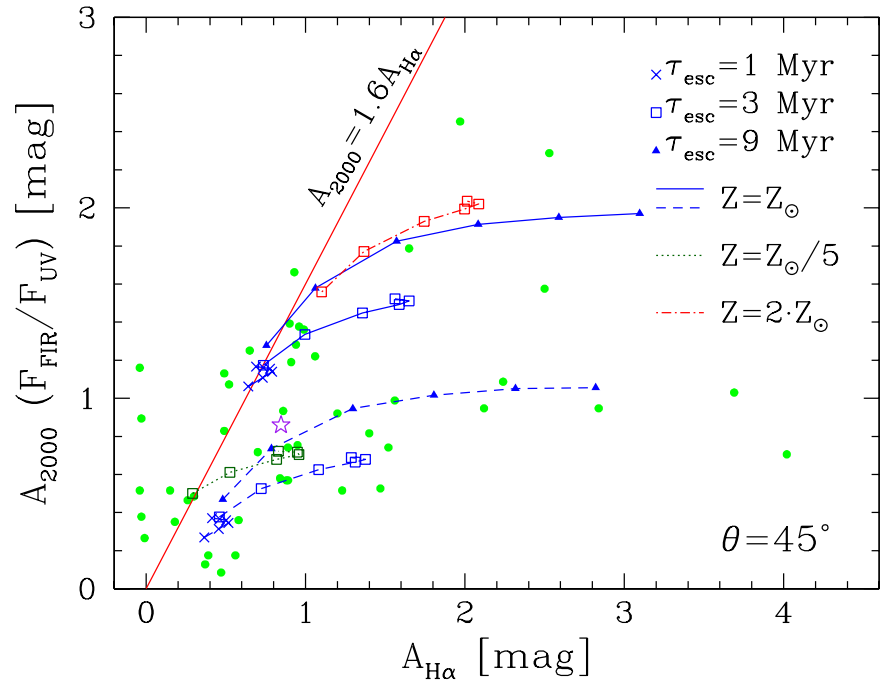

Fig. 5. On the ordinate, $A_{2000}$ derived from the ratio $F_{\mathrm{FIR}} / F_{2000}$ (using Eq. (12)); on the abscissa, $A_{\mathrm{H} \alpha}$ from Balmer decrement. Filled circles show SFG sample data. Lines connect models (see Sect. 4.2) with the same $M_{\mathrm{G}}$ and star formation history, but different $\tau_{\mathrm{MC}}$. Solid lines: $M_{\mathrm{G}}=10^{11} M_{\odot}, v_{\mathrm{sch}}=0.3 \mathrm{Gyr}^{-1}, \tau_{\text {inf }}=18 \mathrm{Gyr}$, solar metallicity; dashed lines: $M_{\mathrm{G}}=10^{10} M_{\odot}, v_{\mathrm{sch}}=0.7 \mathrm{Gyr}^{-1}, \tau_{\mathrm{inf}}=6 \mathrm{Gyr}$, solar metallicity. To emphasize the dependence on metallicity, we show the first model (solid lines) for $\tau_{\text {esc }}=3 \mathrm{Myr}$ but $Z=0.04$ (dot-dashed line) or $Z=0.004$ (dotted line). For simplicity only models with $\theta=45^{\circ}$ are shown here.

where the infrared flux $F_{\text {FIR }}$ is defined as the flux in the [40120] $\mu \mathrm{m}$ interval derived from the 60 and $100 \mu \mathrm{m}$ IRAS bands (Helou et al. 1988), and $F_{1600}=\lambda_{1600} \cdot f_{1600}\left(\right.$ in $\mathrm{W} \mathrm{cm}^{-2}$ ). Note that this relation was derived for starburst galaxies.

In the same framework, Buat et al. (1999) proposed a relation between the $\mathrm{UV}$ attenuation and the $F_{\mathrm{FIR}} / F_{\mathrm{UV}}$ ratio $\left(\lambda_{\mathrm{UV}}=2000 \AA\right)$, which is suited for normal star forming galaxies:

$A_{2000}=0.466+\log \left(\frac{F_{\mathrm{FIR}}}{F_{2000}}\right)+0.433\left[\log \left(\frac{F_{\mathrm{FIR}}}{F_{2000}}\right)\right]^{2}$.

Buat et al. (2002, hereafter B02) compared the $A_{\mathrm{UV}}$ derived from the ratio $F_{\mathrm{FIR}} / F_{\mathrm{UV}}$ (Eq. (12)) with the attenuation suffered by $\mathrm{H} \alpha$ in a sample (called SFG sample) of normal star forming galaxies. The SFG sample consists of 47 spiral and irregular galaxies in nearby clusters. They were observed in the UV $\left(\lambda_{\mathrm{UV}}=2000 \AA\right)$ with the SCAP, FOCA and FAUST instruments (Boselli et al. 2001), in the optical (Gavazzi et al. 2002), and in the FIR by IRAS. The galaxies were selected to have $E W(\mathrm{H} \alpha)>6 \AA$ in order to minimize the errors in $\mathrm{H} \alpha$ and $\mathrm{H} \beta$ fluxes. Galaxies with Seyfert activity were excluded from the sample. Metallicity of the galaxies in the sample ranges from $\sim Z_{\odot} / 4$ to $\sim 2 Z_{\odot}$.

In Fig. 5 we report the attenuation in $\mathrm{H} \alpha$ versus the value of $A_{\mathrm{UV}}$ derived from the ratio $F_{\mathrm{FIR}} / F_{\mathrm{UV}}$ for the SFG sample, as in Fig. 2 of their paper. As pointed out by Buat and collaborators, the two quantities show a lack of correlation, contrary to what is expected from Eq. (9).
Table 1. Values of the relevant parameters used to model normal star forming galaxies. Ref. model: see Sect. 5.

\begin{tabular}{lll}
\hline \hline Param. & All models & Ref. model \\
\hline$\tau_{\text {inf }}$ & $6-18 \mathrm{Gyr}$ & $12 \mathrm{Gyr}$ \\
$v_{\text {sch }}$ & $0.3-0.7 \mathrm{Gyr}^{-1}$ & $0.3 \mathrm{Gyr}^{-1}$ \\
$M_{\mathrm{G}}$ & $10^{10}-10^{11} M_{\odot}$ & $10^{10} M_{\odot}$ \\
$Z$ & $0.004-0.04$ & 0.02 \\
\hline$\tau_{\mathrm{esc}}$ & $1-9 \mathrm{Myr}$ & $3 \mathrm{Myr}$ \\
$\tau_{\mathrm{MC}}$ & $0.1-1.25$ & 0.5 \\
$\theta$ & $0^{\circ}-90^{\circ}$ & \\
$f_{\mathrm{d}}$ & 0.3 & 0.3 \\
\hline
\end{tabular}

\section{2. $A_{U V}$ in simulated normal star-forming galaxies}

In order to give an interpretation of this observational problem, we simulated a set of disk galaxies by exploring the space of parameters appropriate for normal star forming galaxies. We computed the star formation history, gas fraction and metal enrichment with our chemical evolution code (Silva et al. 1998). In order to point out the dependence of the results on metallicity, we considered different values for the metallicity of stars and gas obtained from the chemical code.

The parameters that regulate the star formation history in our models are the baryonic mass of the galaxy $\left(M_{\mathrm{G}}\right)$, the gas infall time scale $\left(\tau_{\text {inf }}\right)$, and the star formation efficiency $v_{\text {sch }}$ of the assumed linear Schmidt law. The age of the galaxies has been set to $10 \mathrm{Gyr}$. The parameters that regulate the attenuation are the escape time $\tau_{\text {esc }}$, the optical thickness of MC at $1 \mu \mathrm{m}$ $\left(\tau_{\mathrm{MC}}\right)$, and the orientation $\theta$ of the disk galaxy with respect to the celestial plane $\left(\theta=0^{\circ}\right.$ means face-on models). The dust (extinction and emission) properties are similar to the galactic one (see Silva et al. 1998 for more details). The dust/gas ratio is assumed to be proportional to the metallicity. Emission lines are computed for different gas densities $n_{\mathrm{H}}$, filling factors $\epsilon$ and stellar masses of clusters $M^{*}$; however we concentrate on $\mathrm{H}$ recombination lines that do not depend on $n_{\mathrm{H}}, M^{*}$ or $\epsilon$. Table 1 summarizes the values of the parameters used in our computations.

The models are compared with data in Fig. 5; the attenuation at $\mathrm{H} \alpha$ is derived from the Balmer decrement (Eq. (10), where we assume $e_{\beta \alpha}=1.47$ ), while the attenuation in UV has been derived by using Eq. (12). For simplicity, we only represent the most dusty (solid lines) and less dusty (dashed lines) models; all other cases range between the two. Models refer to a $45^{\circ}$ inclination; face-on models show a slightly lower attenuation, while edge-on models have larger attenuations and scatter.

The models cover quite well the location of the observed galaxies in this diagram and confirm a real lack of correlation between the UV attenuation, as derived from the $F_{\mathrm{FIR}} / F_{\mathrm{UV}}$ ratio and $A_{\mathrm{H} \alpha}$.

In order to clarify the origin of this scatter and to identify a good estimator of UV attenuation, we contrast the intrinsic 


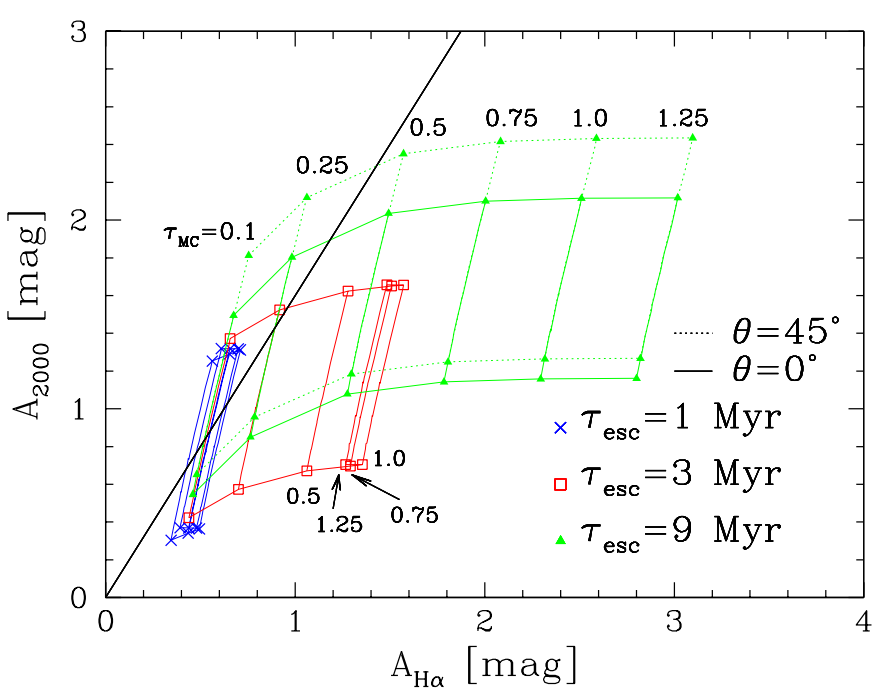

Fig. 6. UV attenuation in the models versus the attenuation at $\mathrm{H} \alpha$ derived from the Balmer decrement. Almost vertical lines connect models with the same optical thickness of $\mathrm{MC}\left(\tau_{\mathrm{MC}}\right)$ and escape time $\left(\tau_{\mathrm{esc}}\right)$, but different SF histories and dust content in cirrus. Only the less and most dusty models are associed to symbols which refer to the escape time. The thick solid line refers to Eq. (9).

UV attenuation - directly extracted from our models $\left(A_{\mathrm{UV}}=\right.$ $\left.-2.5 \log \left(L_{\mathrm{UV}} / L_{\mathrm{UV} 0}\right)\right)$ - respectively with $A_{\mathrm{H} \alpha}$ from the Balmer decrement and $F_{\mathrm{FIR}} / F_{\mathrm{UV}}$ (see Figs. 6 and 7 ).

\subsubsection{Attenuation from Balmer decrement}

The scatter in Fig. 6 must be entirely ascribed to the interplay between the different stellar lifetimes associated to the emission properties and the geometry set by the critical escape time. In fact, $\mathrm{H} \alpha$ is mainly produced by ionizing massive stars with a lifetime around $3 \mathrm{Myr}$, while UV is also emitted by less massive and longer-living stars. We may devise the following typical cases.

Escape time shorter than the typical lifetime of an ionizing star: crosses. Independently of $\tau_{\mathrm{MC}}$, the models tend to define a relation which seems however steeper than Eq. (9). This is due to the fact that both $\mathrm{H} \alpha$ and $\mathrm{UV}$ emissions are mainly produced outside MCs, so that they do not respond to differences in $\tau_{\mathrm{MC}}$ and the attenuation is mainly due to the diffuse medium.

Escape time longer than the typical lifetime of an ionizing star: triangles. Emission lines are produced only inside MCs, so $A_{\mathrm{H} \alpha}$ essentially measures $\tau_{\mathrm{MC}}$. When $\tau_{\mathrm{MC}}>0.5$ the UV flux produced inside MCs is completely reprocessed into the IR. Thus, $A_{\mathrm{Uv}}$ saturates while $A_{\mathrm{H} \alpha}$ still increases. This results in an almost horizontal displacement in Fig. 6.

Escape time comparable to the typical lifetime of an ionizing star: open squares. At increasing $\tau_{\mathrm{MC}}$, the $\mathrm{H} \alpha$ to $\mathrm{H} \beta$ ratio increases from the emitted value to a maximum value fixed by the $\mathrm{H} \beta$ emitted outside the MCs and by the sum of the $\mathrm{H} \alpha$ still coming from within the MCs (the attenuation is lower at $\mathrm{H} \alpha$ than at $\mathrm{H} \beta$ ) and from outside. Then the ratio decreases again to the asymptotic value fixed by the attenuation of the diffuse gas. This causes a behavior that is intermediate between the former two cases and, in particular, gives rise to the turnover shown

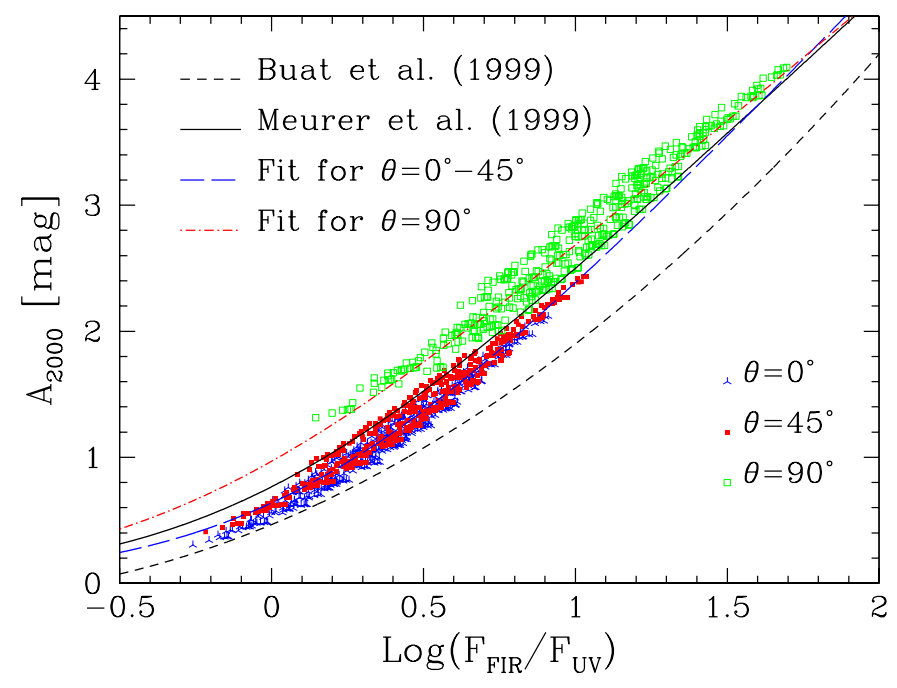

Fig. 7. UV attenuation vs. the ratio $F_{\mathrm{FIR}} / F_{\mathrm{UV}}$; lines refer to Eqs. (11), (12) and our fits.

at high $\tau_{\mathrm{MC}}$. As a consequence, the attenuation in $\mathrm{H} \alpha$ derived from the Balmer decrement is underestimated.

The variation in dust content of the galaxy and/or of the inclination, at constant $\tau_{\mathrm{MC}}$ and $\tau_{\text {esc }}$, shifts the models in Fig. 6 along a constant direction, somewhat steeper than that of Eq. (9); furthermore, a variation in metallicity corresponds to a variation of the amount of dust which varies the attenuation due to the diffuse component. These effects add further dispersion to the data.

Our models suggest that galaxies in Fig. 5 with $A_{\mathrm{H} \alpha} \geq$ 1.5 mag may be characterized by escape times larger than the typical lifetime of the ionizing stars. Models with $A_{\mathrm{H} \alpha} \geq 2.5$ have an equivalent width $E W(\mathrm{H} \alpha)$ lower than $6 \AA$; there are several possible explanations for the higher observed $A_{\mathrm{H} \alpha}$ with large $E W(\mathrm{H} \alpha)$, such as a small burst (increasing the equivalent width), or an underestimate of $\mathrm{H} \beta$ or [N II] lines (giving higher $A_{\mathrm{H} \alpha}$ ).

The results of models and observations described above point out that age selective extinction is present in normal starforming galaxies and complicates the picture of extinction in galaxies. The simple hypothesis of screen extinction does not work in this kind of galaxies; different ways to compute attenuation give inconsistent results because they rise from specific populations (ionizing and non-ionizing UV emitters) that have different lifetimes and live at different optical depths.

\subsubsection{Attenuation from the continuum}

A more robust estimation of the UV attenuation can be obtained by considering the energy reprocessed by dust in the IR.

In Fig. 7 we plot the intrinsic $A_{\mathrm{UV}}$ against the ratio $F_{\mathrm{FIR}} / F_{\mathrm{UV}}$. The solid line represents Eq. (12) and the short dashed line refers to Eq. $(11)^{3}$. Models show that, contrary to the case of Balmer decrement, the UV attenuation correlates

\footnotetext{
3 To convert attenuation at $1600 \AA$ of Eq. (11) into $A_{\mathrm{UV}}$ at $2000 \AA$ we assume $F_{1600}=F_{2000}$ and $A_{2000}=0.9 \cdot A_{1600}=0.9$. $2.5 \log \left(F_{\mathrm{FIR}} / 0.84 F_{2000}+1\right)$, as in $\mathrm{B} 02$.
} 
quite tightly with the $F_{\mathrm{FIR}} / F_{\mathrm{UV}}$ ratio. The origin of this correlation is that the FIR flux is essentially provided by the UV emission of young stellar populations as assumed by Meurer et al. (1999) and Buat et al. (1999). Thus, the $F_{\mathrm{FIR}} / F_{\mathrm{UV}}$ measures the ratio between the reprocessed energy flux and the residual energy flux in the UV. Outside the MCs, a contribution to the FIR comes from the optical emission of old stellar populations; this causes a dispersion around the average relation.

Similar results were obtained by Gordon et al. (2000); their models show that the $A_{\mathrm{UV}}-F_{\mathrm{FIR}} / F_{\mathrm{UV}}$ relationship is valid for different assumptions on dust properties. The dispersion found by Gordon et al. (2000) is much smaller than in our simulations, but in their models all the stellar populations suffer the same extinction (i.e. no dependence on age is introduced).

Models also show that edge-on systems tend to follow a different relation from what is found for face-on systems; this is due to the greater contribution of the diffuse medium to attenuation in the direction of the plane of the galaxy.

Our models suggest the following relation for face-on systems:

$A_{2000}=2.5 \log \left(\frac{F_{\mathrm{FIR}}}{1.25 F_{2000}}+1\right) ;$

while for edge-on systems it is preferable to use

$A_{2000}=2.03 \log \left(\frac{F_{\mathrm{FIR}}}{0.5 F_{2000}}+1\right)$.

In Fig. 7 we also compared the relations proposed by Meurer et al. (1999) (Eq. (11)) and by Buat et al. (1999) (Eq. (12)) with models. The former seems to better agree with our model, and lies between face-on and edge-on models, while the second tends to underestimate the UV attenuation (but only by $0.8 \mathrm{mag}$ in the least favorable case).

Meurer et al. (1999) showed that the observed locus in the diagram $F_{\mathrm{FIR}} / F_{\mathrm{UV}}$ vs. the UV spectral index $\beta$ in starburst galaxies could be well reproduced by a screen dust model with increasing optical thickness. They obtained the following relation between $A_{\mathrm{UV}}$ and $\beta$ :

$A_{1600}=4.43+1.99 \beta$.

Recently, Bell (2002) compared the spectral index $\beta$ with $A_{\mathrm{UV}}(\mathrm{FIR} / \mathrm{UV})$ for a sample of normal star-forming galaxies and found that $\beta$ is typically greater (redder) than the expected value for starbursts.

We also found a loose $\beta-A_{\mathrm{UV}}$ correlation for disk galaxies. Indeed, it is worth noticing that in these galaxies the contribution from stars outside star forming regions is quite substantial and can significantly affect even the UV slope.

\section{SFR estimators}

In the previous section we justified the tangled relation between the attenuation properties of star forming galaxies as seen at different wavelengths, by means of a model that accounts for the complex interplay between geometry, obscuration time and stellar lifetimes.

Using these models, we have obtained calibrations of the SFR for a wide range of different observables from the UV to
Table 2. Calibrations of SFR (SFR/Luminosity) from dust emission, in $10^{-37} M_{\odot} \mathrm{yr}^{-1} \mathrm{~W}^{-1}$ ( or $a: 10^{-33} M_{\odot} \mathrm{yr}^{-1} \mathrm{~W}^{-1} \AA$ ), and from UV luminosity (lower panel, in $10^{-23} M_{\odot} \mathrm{yr}^{-1} \mathrm{~W}^{-1} \mathrm{~Hz}$ ).

\begin{tabular}{lrl}
\hline \hline Band & SFR/L & $\Delta(\mathrm{SFR} / \mathrm{L})$ \\
\hline IR $_{8-1000}$ & 3.99 & $3.12-7.39$ \\
IR & 4.98 & $3.62-8.93$ \\
FIR & 8.82 & $6.12-16.4$ \\
PAH 7.7 $\mu \mathrm{m}$ & 46.7 & $31.3-81.5 a$ \\
ISO LW3 & 79.9 & $70.9-135$. \\
MIPS 24 & 48.0 & $34.6-114$. \\
MIPS 70 & 9.23 & $6.19-17.0$ \\
MIPS 160 & 7.66 & $6.15-13.7$ \\
\hline UV & 284.5 & $103.4-622.8$ \\
UV $_{\beta}$ & 125.9 & $92.7-184.0$ \\
UV $_{\text {FIR }}$ & 104.5 & $90.7-114.5$ \\
\hline
\end{tabular}

the radio regime. Only models with $\tau_{\text {esc }} \leq 6 \mathrm{Myr}$ are used here because the majority of the data in Fig. 5 can be explained with short escape times, $\simeq 3 \mathrm{Myr}$, in agreement with other evidences coming from the analysis of the UV SEDs and from the number counts in HR diagrams of massive stars (Silva et al. 1998). In order to give a useful reference value of the calibration, we selected a reference model whose parameters are summarized in Table 1. This model is represented by a star in Fig. 5. Unless otherwise specified, all calibrations refer to a Salpeter IMF with $m_{\text {inf }}=0.15 M_{\odot}$ and $m_{\text {up }}=120 M_{\odot}$ and solar metallicity.

\subsection{SFR from the continuum}

Infrared luminosity is one of the most common SFR estimators. The interstellar dust is able to convert in IR emission a substantial fraction of the UV light emitted by young stars. Thus, the IR luminosity is proportional to the SFR.

The relation between the SFR and the infrared luminosity is summarized in Table 2. The first column indicates the observed quantity, the second column provides the value of the calibration for the reference model described above, and the last column provides the range of variation of the calibration among the set of models.

The row labeled $\mathrm{IR}_{8-1000}$ refers to the total dust emission from 8 to $1000 \mu \mathrm{m}$, while IR refers to the infrared emission estimated with the four IRAS bands (Sanders \& Mirabel 1996) and FIR refers to the far-infrared emission estimated from the 60 and $100 \mu \mathrm{m}$ IRAS bands (as described in Helou et al. 1988).

In the following rows of Table 2, we show the calibration of the specific luminosity at the peak of the PAH emission feature at $7.7 \mu \mathrm{m}$ (PAH $7.7 \mu \mathrm{m}$ ), the flux in the ISO band LW3, and the SIRTF experiment MIPS at $24 \mu \mathrm{m}, 70 \mu \mathrm{m}$ and $160 \mu \mathrm{m}$.

The infrared estimators exhibit larger variations in normal star-forming galaxies than in starburst galaxies. Inspection of the above table shows that the calibrations can vary by up to $80 \%$ around the reference model. In the case of normal galaxies a considerable part of radiation from young stars is 
Table 3. Calibrations of SFR in UV band in the dust-free case, and for radio emission (in $10^{-23} M_{\odot} \mathrm{yr}^{-1} \mathrm{~W}^{-1} \mathrm{~Hz}$ ) as a function of metallicity.

\begin{tabular}{lrcc}
\hline \hline \multicolumn{1}{c}{$\mathrm{Z}$} & $\mathrm{UV}$ & $1.49 \mathrm{GHz}$ & $8.44 \mathrm{GHz}$ \\
\hline 0.0008 & 78.9 & 59.5 & 166.1 \\
0.004 & 88.7 & 65.1 & 212.6 \\
0.008 & 94.8 & 66.6 & 230.9 \\
0.015 & 99.7 & 67.7 & 246.2 \\
0.02 & 103.4 & 68.6 & 258.4 \\
0.03 & 107.7 & 69.8 & 269.1 \\
0.04 & 112.3 & 71.1 & 280.7 \\
0.05 & 117.4 & 72.5 & 293.5 \\
\hline
\end{tabular}

not absorbed by MCs clouds, so that variations in the duration of the obscuration by MCs and in the optical depth of MCs produce an important change in IR emission. Furthermore, the absorption of light from old populations by diffuse dust may be a significant source of IR radiation, thus weakening the correlation with the SFR. This is quite different from more powerful starbursts where most of the energy produced by young stars is converted into IR emission, so that variations in absorption do not produce any important variation of the IR flux.

The ratio between SFR and UV luminosity $\left(\lambda_{\mathrm{UV}}=2000 \AA\right)$ is presented in the lower panel of Table 2. First row refers to UV uncorrected for dust extinction, the second to the UV luminosity corrected for extinction through the UV slope ( $A_{\mathrm{UV}}$ vs. $\beta$, Eq. (15) converted to $2000 \AA$ ) and the last row to UV luminosity corrected for extinction by adopting the relation $A_{\mathrm{UV}}$ vs. $\log \left(F_{\mathrm{FIR}} / F_{\mathrm{UV}}\right)$ with Eqs. (13) and (14). These calibrations refer to face-on models; calibrations from edge-on models have a much larger range of variation, with the important exception of the FIR-corrected estimator.

It is important to notice that the FIR-corrected UV estimator is quite robust; in fact, as shown in Sect. 4.2, the attenuation in the UV is tightly related to the ratio $F_{\mathrm{FIR}} / F_{\mathrm{UV}}$, for a significant variation of the optical depth and escape time (Fig. 7). Consequently, by adopting the ratio $F_{\text {FIR }} / F_{\text {UV }}$ to estimate $A_{\mathrm{UV}}$, one may get the intrinsic UV flux and obtain a reliable SFR indicator. In summary, UV and FIR fluxes alone are fragile SFR indicators, while their combination is a very good estimator of the UV attenuation. Notice also that, because of the slope of the relation $A_{\mathrm{UV}}$ vs. $F_{\mathrm{FIR}} / F_{\mathrm{UV}}$, this method is not much affected by uncertainties in the FIR flux due to old populations.

We also provide the dust free calibration of UV for different metallicities in Table 3.

Finally, we report in Table 3 the new radio calibrations at $1.49 \mathrm{GHz}$ and $8.44 \mathrm{GHz}$, according to Bressan et al. (2002), as a function of the stellar metallicity. The emission at these frequencies in our model is provided by ionized gas (thermal emission) and SN remnants (non-thermal emission). The variation in luminosity (at constant SFR) is due to the variation of the number of ionizing photons of SSPs with metallicity, of the temperature of the ionized gas for free-free component, and to the variation of $\mathrm{SN}$ rate for non-thermal contributions.
Table 4. Calibrations of SFR for emission lines (in $10^{-33} M_{\odot} \mathrm{yr}^{-1} \mathrm{~W}^{-1}$, or $a$ : in $10^{-35} M_{\odot} \mathrm{yr}^{-1} \mathrm{~W}^{-1}$. No dust: the intrinsic calibrations in the case without dust. Ref. model: calibrations for the reference model. Max value: the maximum value found in our models. The values between brackets in the forth column refer to the edge-on case.

\begin{tabular}{llll}
\hline \hline Line & No dust & Ref. model & Max value \\
\hline $\mathrm{H} \beta$ & 0.187 & 0.758 & $7.94(25.8)$ \\
$\mathrm{H} \alpha_{0}$ & 6.08 & 6.62 & $15.12(29.3) a$ \\
$\mathrm{~Pa} \beta$ & 0.99 & 1.415 & $2.52(4.37)$ \\
$\mathrm{Pa} \beta_{0}$ & 0.99 & 0.99 & $1.06(1.18)$ \\
$\mathrm{Pa} \alpha$ & 0.436 & 0.505 & $0.676(0.938)$ \\
$\mathrm{Br} \gamma$ & 5.69 & 6.31 & $7.86(10.2)$ \\
$\mathrm{Br} \beta$ & 3.28 & 3.50 & $4.12(4.96)$ \\
$\mathrm{Br} \alpha$ & 1.69 & 1.721 & $1.91(2.10)$ \\
$\mathrm{Hu} \alpha$ & 12.9 & 13.12 & $14.7(16.2)$ \\
{$[\mathrm{O} \mathrm{II}] 3727$} & 0.127 & 0.844 & $25.2(104.8)$ \\
\hline
\end{tabular}

It is worth noticing that the variation of $1.49 \mathrm{GHz}$ calibration with metallicity is $\pm 15 \%$.

Observations indicate that FIR and radio emissions are strongly correlated over a wide range of IR luminosities, from star-forming to starburst galaxies (Sanders \& Mirabel 1996):

$q=\log \frac{F_{\mathrm{FIR}} /\left(3.75 \times 10^{12} \mathrm{~Hz}\right)}{F_{1.49 \mathrm{GHz}} /\left(\mathrm{W} \mathrm{m}^{-2} \mathrm{~Hz}^{-1}\right)} \simeq 2.35 \pm 0.2$.

From calibrations in Table 2 and in Table 3, for the FIR and the $1.49 \mathrm{GHz}$, we obtain $q=2.32$ for the reference model, with a range of variation $q=2.05-2.48$.

Remarkably, the 0.2 dex observed scatter is fully accounted in our models simply by the variation of the SFR-FIR relationship (see also Perez-Olea \& Colina 1995). This suggests that the relationship between radio emission and star formation is extremely tight and implies that the underlying physical mechanism responsible for it is quite homogeneous.

Although IR and radio calibrations depend on $f_{\mathrm{d}}$ (here assumed to be 0.3$)$, their values change by less than $5 \%(25 \%$ for $8.44 \mathrm{GHz}$ ) if $f_{\mathrm{d}}=0$ is assumed.

\subsection{Optical near- and mid-infrared emission lines}

$\mathrm{H}$ recombination lines are the only ones almost proportional to the Lyman continuum flux and, as a consequence, to the SFR; other emission lines also depend on other quantities so that their use as SFR estimators is quite dangerous.

In Table 4 we report our calibrations for some emission lines. The second column shows the intrinsic calibrations (without dust); the third column provides the calibration for the reference model described above. The forth column provides the maximum value found in our models (the minimum value of calibrations corresponds to the case without dust).

The calibrations are given assuming that the fraction of ionizing photons absorbed by dust inside $\mathrm{H}$ II regions $\left(f_{\mathrm{d}}\right)$ is 0 . 
Calibrations for different values $f_{\mathrm{d}}$ can be obtained by dividing the value reported by $1-f_{\mathrm{d}}$.

$\mathrm{H} \beta$. The first entry in the table is $\mathrm{H} \beta$ uncorrected for extinction; this line may be the only hydrogen line detected in the spectra of intermediate-redshift galaxies and, in that case there is no way to correct it for extinction. The calibration we provide here shows that for a typical SFR of a few $M_{\odot} / y r$, the inferred SFR may be underestimated by a factor of three.

$\mathrm{H} \alpha_{0}$. The $\mathrm{H} \alpha_{0}$ row refers to the $\mathrm{H} \alpha$ luminosity corrected for extinction using the Balmer decrement (Eq. (10)). We notice that the decrement has been evaluated from comparisons of the observed ratio of the intensity of the emissions at $\mathrm{H} \alpha$ and $\mathrm{H} \beta$ with the expected ratio. We did not attempt to simulate a real measure of the Balmer decrement in a synthetic spectrum. Thus, our calibration assumes that one is able to correct the lines for the contribution of the underlying older populations.

It is worth noticing that, due to the age selective extinction, the calibration may change by a factor of two, or even more because the extinction at $\mathrm{H} \alpha$ is underestimated, as demonstrated in Sect. 4.2.1, when the escape time is similar to the lifetime of ionizing stars.

Another factor of uncertainty is provided by the orientation of the observed galaxy because the extinction from diffuse medium is characterized by a mixed geometry, which tends to produce a higher ratio between attenuation and reddening than for the screen geometry.

$\mathrm{Pa} \beta, \mathrm{Pa} \beta_{0}$. In Table 4 then $\mathrm{Pa} \beta$ follows, either uncorrected or corrected $\left(\mathrm{Pa} \beta_{0}\right)$ for extinction, assuming that the intrinsic value of $\mathrm{Pa} \beta / \mathrm{Br} \gamma$ is 5.65 (and $\tau_{\mathrm{Pa} \beta} / \tau_{\mathrm{Br} \gamma}=2.95$ ). In this case the extinction is lower and the SFR can be obtained with a large accuracy. However, it must be kept in mind that at these wavelengths uncertainties in the photometry of different wavebands may constitute the larger source of uncertainty in the estimated extinction (e.g. Calzetti et al. 1996).

$\mathrm{Pa} \alpha$. The table continues with $\mathrm{Pa} \alpha$ uncorrected for extinction. This line sits at the short wavelength border of the $K$ band $(\lambda=18752 \AA)$; like $\mathrm{H} \beta$, it may be detected by ground observations only in the spectra of intermediate redshift galaxies.

$\operatorname{Br} \gamma, \operatorname{Br} \beta, \operatorname{Br} \alpha, \operatorname{Hu} \alpha$. The following entries in the table are $\operatorname{Br} \gamma, \operatorname{Br} \beta, \operatorname{Br} \alpha$ and $\mathrm{Hu} \alpha$ uncorrected for extinction. As expected, the extinction effects decrease when the wavelength increases, except for $\operatorname{Hu} \alpha(\lambda=12.371 \mu \mathrm{m})$ that falls in the absorption feature of silicates.

[O II $] 3727$. The table closes with the [O II]3727 line uncorrected for extinction (note we only use solar metallicity). The importance of this line is that in high redshift galaxies this is the only bright line that, once redshifted, remains in the optical domain. Recently, Jansen et al. (2001) investigated the use of this line as SFR tracer, and found that SFR estimations by means of [O II]3727 agree with the $\mathrm{H} \alpha_{0}$ value, as long as the line intensity is corrected for extinction and calibrated for metallicity. However, it is not possible to perform these corrections without observing other lines that (if visible) are better estimators of SFR; therefore we conclude that [O II] 3727 is a poor indicator of SFR.

\section{Infrared nebular metallic lines}

The IR spectral range contains numerous bright forbidden finestructure lines that are excellent diagnostics of gas density, hardness of the exciting radiation field and abundance of important elements.

\subsection{Density and ionizing spectrum hardness diagniostics}

Different IR fine structure transitions of the same ion have different sensitivity to collisional deexcitation. This can be used to identify the typical electron density of the emitting gas (Rubin et al. 1994). Typical line pairs used for this purpose are [Ar III] $21.8 \mu \mathrm{m} / 9 \mu \mathrm{m}$, with a maximum sensitivity to the electron density at $\log \left(n_{\mathrm{e}}\right)=4.7,[\mathrm{Ne} \mathrm{III}] 36 / 15.5 \mu \mathrm{m}\left(\log \left(n_{\mathrm{e}}\right)=\right.$ 4.7), [O III]52/88 $\mu \mathrm{m}\left(\log \left(n_{\mathrm{e}}\right)=2.9\right),[\mathrm{S}$ III] $19 / 33 \mu \mathrm{m}$ $\left(\log \left(n_{\mathrm{e}}\right)=3.5\right)$, and $[\mathrm{N}$ II $] 122 / 205 \mu \mathrm{m}\left(\log \left(n_{\mathrm{e}}\right)=1.8\right)$. Though collision strengths for these lines may need to be reexamined (Rubin et al. 2001), it appears that they can trace the density of the emitting gas over a wide range, from $n_{\mathrm{H}}=10 \mathrm{~cm}^{-3}$ to $n_{\mathrm{H}}=10^{5} \mathrm{~cm}^{-3}$, i.e. possibly encompassing the typical densities of star forming regions in normal galaxies as well as in compact obscured starbursts (Bressan et al. 2002).

The hardness of the ionizing spectrum and the average ionization parameter may be derived by analyzing ratios of line intensities in different ionization stages of suitable elements. Typical ratios are [Ne III]/[Ne II], [Ar III]/[Ar II] and [S IV]/[S III] (e.g. Giveon et al. 2002). Our model can be used to obtain information on the age of the ionizing stellar population and on the ionization parameter in single $\mathrm{H}$ II regions or in starbursts of short duration. In other conditions, this method will only provide the average values for these quantities. We will explore these topics in a forthcoming paper.

\subsection{Abundance determinations}

It is straightforward to extend to the infrared the method used for abundance determinations for optical lines. This method is based on the ratio between the sum of the intensities of the most abundant ions of an element, and that of a hydrogen recombination line (typically the $\operatorname{Br} \alpha$ line). It works as long as the current density is lower than the critical density for collisional deexcitation.

In the near- and mid-infrared the elements to which we can apply the above method are neon ([Ne III]15.5 $\mu \mathrm{m}$ and $[\mathrm{Ne} \mathrm{II}] 12.8 \mu \mathrm{m})$, argon ([ArIII]9 $\mu \mathrm{m}$ and $[\mathrm{ArII}] 7 \mu \mathrm{m})$ and sulphur ([S IV]10.5 $\mu \mathrm{m},[\mathrm{S}$ III] $18.7 \mu \mathrm{m}$ and [S II] $1.03 \mu \mathrm{m}$ ). Suitable calibrating relationships can be found in Giveon et al. (2002) and Verma et al. (2003).

In the far-infrared the method is applicable only to nitrogen $([\mathrm{N}$ II $] 122 \mu \mathrm{m}$ and $[\mathrm{N}$ III $] 57 \mu \mathrm{m})$. For these lines we have 


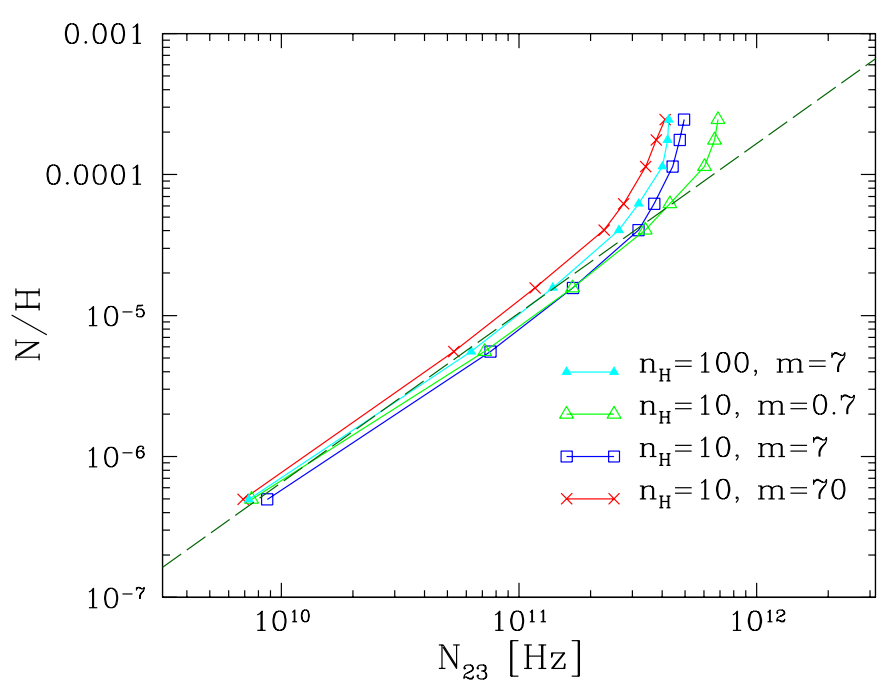

Fig. 8. The relationship between nitrogen abundance (relative to hydrogen) and $N_{23}$ (defined by Eq. (18)) for models with different densities and ionization parameters. The dashed line shows a linear fit to models with $Z<0.015$.

derived the following calibrations with respect to the $\operatorname{Br} \alpha$ line (for different assumptions on the electronic temperature $T_{\mathrm{e}}$ ):

$\frac{\mathrm{N}}{\mathrm{H}}=\left\{\begin{array}{r}1.907 \times 10^{-5}\left(L_{[\mathrm{NII}] 122}+0.247 L_{[\mathrm{N} I I] 57}\right) / L_{\mathrm{Br} \alpha} \\ \text { for } T_{\mathrm{e}}=10000 \mathrm{~K} \\ \\ 3.546 \times 10^{-5}\left(L_{[\mathrm{NII}] 122}+0.231 L_{[\mathrm{N} I I] 57}\right) / L_{\mathrm{Br} \alpha} \\ \text { for } T_{\mathrm{e}}=5000 \mathrm{~K}\end{array}\right.$

where $\mathrm{N} / \mathrm{H}$ is the nitrogen abundance respect to hydrogen. The relevant transition data have been taken from Lennon \& Burke (1994), Peng \& Pradhan (1995) and Storey \& Hummer (1995).

It is worth noticing that this method requires the measurement of the intrinsic intensity of a hydrogen recombination line. Thus, it is not applicable when these lines are not accessible, either because of instrumental limitation or for very high extinction. In this case, we suggest to adopt another indicator tightly correlated with the intrinsic intensity of hydrogen recombination lines or, equivalently, with the SFR. The best SFR indicator we have found is the radio luminosity (Table 3). To this purpose, we have run a set of models varying the metallicity of the gas (from $Z=0.0008$ to $Z=0.05$ ), the hydrogen density, from 10 to $100 \mathrm{~cm}^{-3}$, and the ionization parameter. The latter is a function of the mass of ionizing clusters $M^{*}$, and of the filling factor $\epsilon$ through Eq. (8). We stress that the emission lines resulting from quiescent star forming galaxies are the sum of the emission of H II regions of different ages, so that there is not a single ionization parameter $U$ at work. However, since all the ionization parameters scale linearly with the quantity $m \equiv\left(M^{*} \epsilon^{2}\right)^{1 / 3}$, we have changed $m$ from 0.7 to $70 M_{\odot}^{1 / 3}$ to simulate a similar range of variation of $U$. The fraction of ionizing photon absorbed by dust was assumed to be $f_{\mathrm{d}}=0.3$.

Figure 8 shows the relationship in our models between the nitrogen abundance and the ratio $N_{23}$ defined as

$N_{23}=\left(L_{[\mathrm{N} \text { II }] 122}+0.247 L_{[\mathrm{N} \text { III }] 57}\right) / L_{1.49 \mathrm{GHz}} \cdot$
We notice that the metallicity is well determined for values below solar. For higher values the relation steepens considerably making the determination of the metal content quite uncertain.

A linear fit to models in which the metallicity is lower than 0.015 is plotted in Fig. 8; its analytical expression is

$\log \left(\frac{\mathrm{N}}{\mathrm{H}}\right)=-18.21+1.203 \log N_{23}$,

where $N_{23}$ is given in hertz.

We stress that this relation holds as long as the density is below the critical density for collisional de-excitation of the [N II] $122 \mu \mathrm{m}$ transition $\left(n_{\mathrm{e}} \sim 300 \mathrm{~cm}^{-3}\right)$.

A similar calibration could be obtained by adopting the FIR luminosity instead of the radio luminosity. However, in the case of disk galaxies the relation between the FIR luminosity and the SFR is not as tight as that between the radio luminosity and the SFR (see Table 2). This would introduce a significant scatter in the calibration.

\section{Discussion}

The first application of the model presented in this work is the analysis of attenuation in quiescent star-forming galaxies.

These galaxies exhibit a poor correlation between the attenuation in the UV band and $\mathrm{H} \alpha$ (B02, see also Fig. 5), with the former generally lower than what expected from the latter on the basis of the simple law $A_{\mathrm{UV}}=1.6 A_{\mathrm{H} \alpha}$ (Eq. (9)). Our model explains this poor correlation in the context of age selective extinction. As the extinction in the UV is very high, the contribution to the observed UV flux from stars outside the molecular clouds is important even for relatively small optical thicknesses $\left(\tau_{\mathrm{MC}}\right)$. It is worth noticing that the fraction of young UV emitting stars outside MCs increases for decreasing escape time, while very young stars (which ionize the gas) spend most of their life inside MCs. Therefore, above a threshold value for $\tau_{\mathrm{MC}}$ which depends on the escape time, an increase of $\tau_{\mathrm{MC}}$ produces an attenuation in $\mathrm{H} \alpha$ larger than that in UV.

Thus, neither observations nor modeling support the assumption of a constant relationship between the attenuation suffered by the continuum and the attenuation for the gas, at least for a current SFR smaller than $10 M_{\odot} / \mathrm{yr}$. As a consequence, the results of Calzetti (1997) $\left(A_{6563}^{\text {stars }} / A_{\mathrm{H} \alpha}^{\mathrm{gas}} \sim 0.44\right)$ cannot be extrapolated from starbursts to disk star-forming galaxies.

Moreover, the extrapolation of the $\mathrm{H} \alpha$ attenuation to the UV through the previous reported simple law yields UV corrected fluxes larger than those expected from $\mathrm{H} \alpha$ corrected fluxes, as found by Sullivan et al. (2000, 2001). These authors explain the result by introducing star formation histories that change rapidly with time and/or a more complex model of extinction. On the other hand, our model explains this result as a natural consequence of the age selective extinction.

An additional interesting issue is that the attenuation in $\mathrm{H} \alpha$ derived from the Balmer decrement can be underestimated, since in disk galaxies the escape time and the lifetime of very massive stars is often similar.

The comprehensive treatment of nebular and continuum emission allows to conclude that the assumption of a constant 
Table 5. Comparison of some calibrations of SFR with results from previous works. Calibrations are referred to a Salpeter IMF between 0.1 and $100 M_{\odot}$. Reference: $a$ : Kennicutt (1998), $b$ : Haarsma et al. (2000).

\begin{tabular}{llll}
\hline \hline & This work & others & \\
$\mathrm{UV}$ & 1.20 & $1.4 \times 10^{-21} M_{\odot} \mathrm{yr}^{-1} \mathrm{~W}^{-1} \mathrm{~Hz}$ & $a$ \\
$\mathrm{IR}_{8-1000}$ & 4.63 & $4.5 \times 10^{-37} M_{\odot} \mathrm{yr}^{-1} \mathrm{~W}^{-1}$ & $a$ \\
$\mathrm{H} \alpha$ & 7.05 & $7.9 \times 10^{-35} M_{\odot} \mathrm{yr}^{-1} \mathrm{~W}^{-1}$ & $a$ \\
$1.49 \mathrm{GHz}$ & 79.6 & $123.3 \times 10^{-23} M_{\odot} \mathrm{yr}^{-1} \mathrm{~W}^{-1} \mathrm{~Hz}$ & $b$ \\
\hline
\end{tabular}

extinction for all stellar populations is not satisfactory. However, the detailed models presented in this work indicate that $A_{\mathrm{UV}}$ can be accurately estimated by using the ratio $F_{\mathrm{FIR}} / F_{\mathrm{UV}}$ (cf. Eqs. (13) and (14)). This conclusion holds provided that the main contribution to FIR comes from the absorbed UV radiation of young stars, but it does not depend on the escape times and on the extinction properties of dust. The relationships we obtain are intermediate between those by Buat et al. (1999) and by Meurer et al. (1999), the latter derived for starburst galaxies.

By using our set of simulated disk galaxies, we calibrated different SFR estimators, collected in Tables 2-4. Some calibrations may be compared with values obtained in the literature. In particular we compare the results with Kennicutt (1998) (UV, IR and $\mathrm{H} \alpha$ ) and Haarsma et al. (2000) (radio); these comparisons are summarized in Table 5, after a small correction factor (1.16), which accounts for the slightly different IMF adopted, is applied to our estimates.

There is a good agreement for $\mathrm{H} \alpha$, UV and IR calibrations (note that Kennicutt 1998 uses the notation FIR for the total dust emission between 8 and $1000 \mu \mathrm{m}$ ), while our calibration for the radio emission is a factor 1.5 smaller than that proposed by Condon (1992) and Haarsma et al. (2000), and a factor 1.3 larger than the one quoted by Carilli (2002).

It is worth noticing that our calibrations give a ratio (Eq. (16)) between the radio and FIR emissions, $q=2.32$, very close to the observed value, and that we can explain in a natural way the scatter around the observed relation. In disk galaxies, this scatter is due to the variation of the fraction of stellar emission absorbed (and re-radiated) by dust.

In the case of normal star forming galaxies, the model evidences that $\mathrm{H} \alpha$, UV and even IR estimators of SFR are affected by important scatters. For what concerns $\mathrm{H} \alpha$, this is due to the age selective extinction, that can produce an underestimate of the attenuation when it is derived by the Balmer decrement. Also IR emission by itself does not provide an accurate estimate of the SFR in disk galaxies, because the fraction of UV radiation not absorbed by the dust can be high. The combination of UV and FIR luminosities provides a very good SFR estimator essentially because, even for a modest SFR, one recovers in the FIR what is lost in the UV.

Finally we have also discussed the utility of IR nebular lines as diagnostic tools for deriving the average properties of the environment such as number density, hardness of ionizing spectrum and gas metallicity. In particular we have provided a new calibration for the nitrogen abundance $(\mathrm{N} / \mathrm{H})$ as a function of the intensities of the [N II] $122 \mu \mathrm{m}$ and [N III] $57 \mu \mathrm{m}$, and $\operatorname{Br} \alpha$ lines. When the latter line is missing or useless (e.g. when affected by strong extinction), we suggest the possible use of the radio luminosity as indicator of the ionizing flux $Q_{\mathrm{H}}$, that enters implicitely the above calibration. We provide a new calibration of the metallicity with the ratio $N_{23}$ defined by Eq. (18). This new calibration will turn out to be particularly useful for the Herschel experiment.

\section{Summary}

Here we summarize the main points of this paper.

1. We have introduced the nebular emission calculations from CLOUDY into our spectrophotometric code GRASIL. The method which interfaces nebular emission computations with population synthesis is based on pre-computed libraries of H II region models. These libraries can be retrieved from the GRASIL web site ${ }^{4}$ and easily used for other population synthesis codes.

2. As a result, we can model the spectra of star-forming galaxies from far-UV to radio wavelengths, including stellar absorption features, nebular emission, dust and PAH emission.

3. We applied the model to study the attenuation in normal star forming galaxies. The poor correlation between the extinction at UV and $\mathrm{H} \alpha$ found by B02 is interpreted in our model as a natural consequence of age selective extinction.

4. Conversely, we found that $A_{\mathrm{UV}}$ correlates quite tightly with the ratio $F_{\mathrm{FIR}} / F_{\mathrm{UV}}$.

5. We present new calibrations for the SFR using lines and continuum from UV to radio wavelengths, and study the reliability of each estimator. In particular, NIR $\mathrm{H}$ recombination lines and radio luminosities are very accurate SFR estimators.

6. Although UV-, $\mathrm{H} \alpha-$ (also corrected for extinction) and IR-derived SFR are plagued by significant uncertainties, the UV luminosity corrected by using the ratio $F_{\mathrm{FIR}} / F_{\mathrm{UV}}$ is a robust SFR estimator.

7. We reproduce the observed value of the $q$ ratio between radio and FIR emission. Its scatter is ascribed to the variation of extinction between different objects.

8. Finally, we discuss several methods and provide new calibrations for the determination of metal abundances by means of infrared emission lines.

Acknowledgements. We thank A. Boselli, V. Buat and M. Magliocchetti for enlightening discussions, and the referee (M. Fioc) for the very accurate scrutiny of the paper and useful suggestions. A. B. acknowledges warm hospitality by INAOE (MEX). We are also grateful to G. J. Ferland for the public access to CLOUDY. This research was partially supported by the Italian Ministry for University and Research (MIUR) and ASI.

\footnotetext{
${ }^{4}$ http://web.pd.astro.it/granato/grasil/grasil.html
} 


\section{Appendix A: Analytical spectra}

Here we present the relationships used to describe the parameters of analytical spectra introduced in Eq. (1) as functions of $Q_{\mathrm{H}}, Q_{\mathrm{He}}$, and $Q_{\mathrm{O}}$.

$T_{\mathrm{He}}$ is implicitly given by:

$\frac{\int_{v_{\mathrm{O}}}^{\infty} F_{\nu} \mathrm{d} v / h v}{\int_{v_{\mathrm{He}}}^{\infty} F_{\nu} \mathrm{d} v / h v}=\frac{\int_{v_{\mathrm{O}}}^{\nu_{\mathrm{He}^{+}}} B_{\nu}\left(T_{\mathrm{He}}\right) \mathrm{d} v / h v}{\int_{\nu_{\mathrm{He}}}^{v_{\mathrm{He}^{+}}} B_{v}\left(T_{\mathrm{He}}\right) \mathrm{d} v / h v}=\frac{Q_{\mathrm{O}}}{Q_{\mathrm{He}}}$,

where $v_{\mathrm{He}^{+}}$is the photoionization threshold frequency for He II. Once $T_{\mathrm{He}}$ is known, $A_{\mathrm{He}}$ is obtained from

$A_{\mathrm{He}} \int_{v_{\mathrm{He}}}^{v_{\mathrm{He}^{+}}} \frac{B_{v}\left(T_{\mathrm{He}}\right)}{h v} \mathrm{~d} v=Q_{\mathrm{He}}$.

Furthermore, to compute $T_{\mathrm{H}}$, we notice that there exists (for a wide range of age and metallicities) a correlation between $T_{\mathrm{H}}$ and $Q_{\mathrm{He}} / Q_{\mathrm{H}}$ of the SSP spectra, (Fig. A.1):

$T_{\mathrm{H}}=\left\{\begin{array}{ll}3 \times 10^{4}+4 \times 10^{4} \frac{Q_{\mathrm{He}}}{Q_{\mathrm{H}}} & \text { for } \frac{Q_{\mathrm{He}}}{Q_{\mathrm{H}}}>0.005 \\ 4 \times 10^{4}+0.5 \times 10^{4} \cdot \log \left(\frac{Q_{\mathrm{He}}}{Q_{\mathrm{H}}}\right) & \text { for } \frac{Q_{\mathrm{He}}}{Q_{\mathrm{H}}}<0.005\end{array}\right.$.

We use this equation to derive $T_{\mathrm{H}}$ for given values of $Q_{\mathrm{He}} / Q_{\mathrm{H}}$. Then $A_{\mathrm{H}}$ follows from

$A_{\mathrm{H}} \int_{v_{\mathrm{H}}}^{v_{\mathrm{He}}} \frac{B_{\nu}\left(T_{\mathrm{H}}\right)}{h v} \mathrm{~d} v=Q_{\mathrm{H}}-Q_{\mathrm{He}}$.

As for the values of $A_{\mathrm{ni}}$ and of the temperature $T_{\mathrm{ni}}$, we notice that the region of the spectrum with $\lambda>912 \AA$ becomes important only for relatively low ionizing fluxes. We thus simply obtained their values after minimization of the differences between the lines computed with full SSPs spectra and analytical spectra:

$T_{\mathrm{ni}}=\left\{\begin{array}{ll}\operatorname{Max}\left(T_{\mathrm{H}}, 40000 \mathrm{~K}\right) & \text { for } Q_{\mathrm{He}} / Q_{\mathrm{H}}>0.005 \\ 30000 \mathrm{~K} & \text { for } Q_{\mathrm{He}} / Q_{\mathrm{H}}<0.005\end{array}\right.$,

$A_{\text {ni }}=\left\{\begin{array}{ll}2.5 A_{\mathrm{H}} & \text { for } Q_{\mathrm{He}} / Q_{\mathrm{H}}>0.005 \\ 3.5 A_{\mathrm{H}} & \text { for } Q_{\mathrm{He}} / Q_{\mathrm{H}}<0.005\end{array}\right.$.

In Fig. A. 2 we compare some SSP spectra with the corresponding analytical spectra. These comparisons suggest that analytical spectra can represent the Lyman continuum with reasonable precision. However, we stress that our goal is to get line emissions computed with analytical spectra which are as similar as possible to the ones computed with SSP.

\section{Appendix B: Average ionization parameter for a sphere}

The volume averaged ionization parameter for a shere is defined as:

$\langle U\rangle=\int_{0}^{R_{\mathrm{S}}} \frac{U(r) 4 \pi r^{2} \mathrm{~d} r}{\frac{4 \pi}{3} R_{\mathrm{S}}^{3}}$,

where $R_{\mathrm{S}}$ is the Strömgren radius and $U(r)$ is the ionization parameter of the gas at the distance $r$ from the source. $U(r)$ is defined by:

$U(r)=\frac{Q(r)}{4 \pi r^{2} n_{\mathrm{H}} c}$,

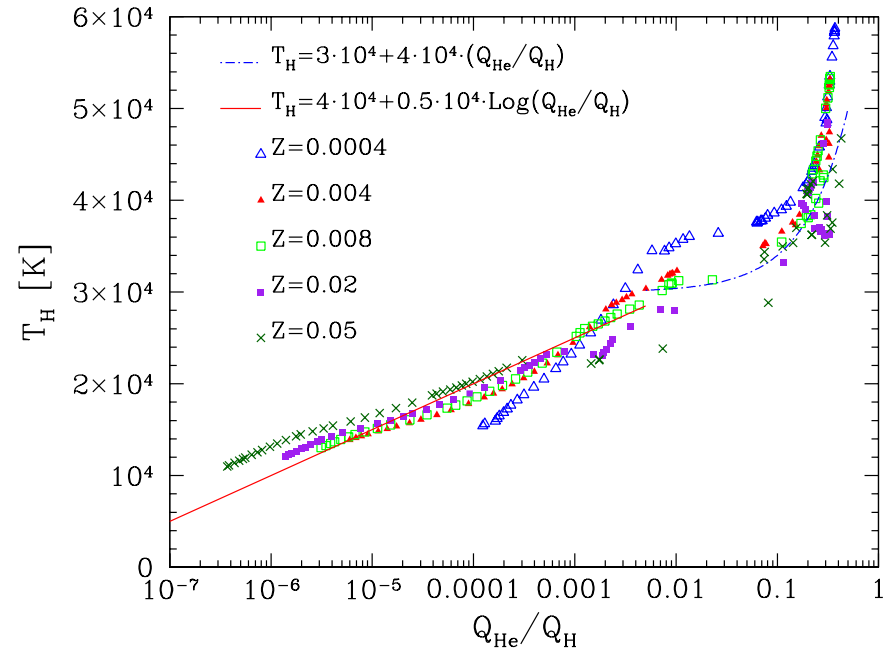

Fig. A.1. Temperature of ionizing continuum $T_{\mathrm{H}}$ as a function of $Q_{\mathrm{He}} / Q_{\mathrm{H}}$ for SSPs of different age and metallicity (points). The lines refer to the analytical fit (Eq. (A.3)).

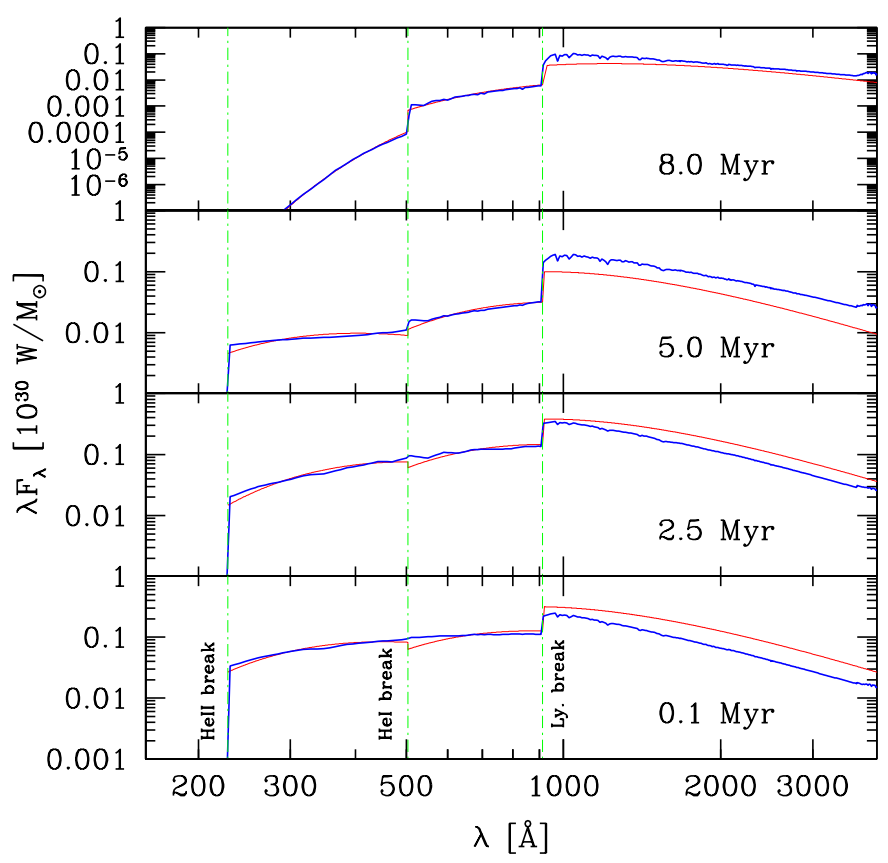

Fig. A.2. UV and ionizing spectra for SSPs $(Z=0.008)$ of different ages (thick lines) compared with the corresponding analytical spectra (thin lines) that have the same $Q$ values than the SSP spectra.

where $Q(r)$ is the number of ionizing photons per unit time that cross a spherical surphace at distance $r$. The variation of $Q(r)$ with the radius is given by:

$\frac{\mathrm{d} Q}{\mathrm{~d} r}=-4 \pi r^{2} n_{\mathrm{H}}^{2} \alpha_{\mathrm{B}}(\mathrm{H}) \epsilon$.

Integrating this equation we get:

$Q(r)=Q_{\mathrm{H}}-\frac{4 \pi}{3} r^{3} n_{\mathrm{H}}^{2} \alpha_{\mathrm{B}}(\mathrm{H}) \epsilon$.

The Strömgren radius is defined as:

$R_{\mathrm{S}}=\left(\frac{3 Q_{\mathrm{H}}}{4 \pi n_{\mathrm{H}}^{2} \alpha_{\mathrm{B}}(\mathrm{H}) \epsilon}\right)^{\frac{1}{3}}$. 
By substituting Eqs. (B.4) and (B.5) in Eq. (B.1), we get:

$$
\begin{aligned}
\langle U\rangle= & \int_{0}^{R_{\mathrm{S}}}\left(\frac{Q_{\mathrm{H}}}{4 \pi r^{2} n_{\mathrm{H}} c}-\frac{r n_{\mathrm{H}} \alpha_{\mathrm{B}}(\mathrm{H}) \epsilon}{3 c}\right) \frac{3 r^{2} \mathrm{~d} r}{R_{\mathrm{S}}^{3}} \\
= & \frac{3 Q_{\mathrm{H}}}{4 \pi n_{\mathrm{H}} c}\left(\frac{4 \pi n_{\mathrm{H}}^{2} \alpha_{\mathrm{B}}(\mathrm{H}) \epsilon}{3 Q_{\mathrm{H}}}\right)^{\frac{2}{3}} \\
& -\frac{n_{\mathrm{H}} \alpha_{\mathrm{B}}(\mathrm{H}) \epsilon}{4 c}\left(\frac{3 Q_{\mathrm{H}}}{4 \pi n_{\mathrm{H}}^{2} \alpha_{\mathrm{B}}(\mathrm{H}) \epsilon}\right)^{\frac{1}{3}} \\
= & \frac{3}{4 c}\left(\frac{3 Q_{\mathrm{H}} \alpha_{\mathrm{B}}^{2}(\mathrm{H}) n_{\mathrm{H}} \epsilon^{2}}{4 \pi}\right)^{\frac{1}{3}} .
\end{aligned}
$$

\section{References}

Aller, L. H. 1984, Physics of Thermal Gaseous Nebulae, Astrophysics and Space Science Library (Boston: Reidel)

Bell, E. F. 2002, ApJ, 577, 150

Binette, L., Magris, C. G., Stasińska, G., \& Bruzual, A. G. 1994, A\&A, 292, 13

Boselli, A., Gavazzi, G., Donas, J., \& Scodeggio, M. 2001, AJ, 121, 753

Bressan, A., Chiosi, C., \& Fagotto, F. 1994, ApJS, 94, 63

Bressan, A., Silva, L., \& Granato, G. L. 2002, A\&A, 392, 377

Buat, V., Donas, J., Millard, B., \& Xu, C. 1999, A\&A, 352, 371

Buat, V., Boselli, A., Gavazzi, G., \& Bonfanti, C. 2002, A\&A, 383, 801 (B02)

Burgess, A., \& Summers, H. P. 1976, MNRAS, 174, 345

Calzetti, D. 1997, AJ, 113, 162

Calzetti, D., Kinney, A. L., \& Storchi-Bergmann, T. 1994, ApJ, 429, 582

Calzetti, D., Kinney, A. L., \& Storchi-Bergmann, T. 1996, ApJ, 458, 132

Calzetti, D., Armus, L., Bohlin, R. C., et al. 2000, ApJ, 533, 682

Carilli, C. L., 2002, Am. Astron. Soc., 200, 5113 [astro-ph/0011199]

Charlot, S., \& Fall, S. M. 2000, ApJ, 539, 718

Charlot, S., \& Longhetti, M. 2001, MNRAS, 323, 887 (CL01)

Condon, J. J. 1992, ARA\&A, 30, 575

Conti, P. S. 1991, ApJ, 377, 115

DeGioia-Eastwood, K. 1992, ApJ, 397, 542

Dessauges-Zavadsky, M., Pindao, M., Maeder, A., \& Kunth, D. 2000, A\&A, 355, 89

Ferland, G. J. 1980, PASP, 92, 596

Ferland, G. J. 2001, Hazy, a brief introduction to CLOUDY, University of Kentucky, Department of Physics and Astronomy Internal Report

Fioc, M., \& Rocca-Volmerange, B. 1997, A\&A, 326, 950

Franceschini, A., Silva, L., Fasano, G., et al. 1998, ApJ , 506, 600

Fuente, A., Neri, R., Martín-Pintado, J., et al. 2001, A\&A, 366, 873

Gavazzi, G., Bonfanti, C., Sanvito, G., Boselli, A., \& Scodeggio, M. 2002, ApJ, 576, 135

Giveon, U., Sternberg, A., Lutz, D., Feuchtgruber, H., \& Pauldrach, A. W. A. 2002, ApJ, 566, 880

Granato, G. L., Lacey, C. G., Silva, L., et al. 2000, ApJ, 542, 710

Granato, G. L., Silva, L., Monaco, P., et al. 2001, MNRAS, 324, 757

Gordon, K. D., Clayton, G. C., Witt, A. N., \& Misselt, K. A. 2000, ApJ, 533, 236

Haarsma, D. B., Partridge, R. B., Windhorst, R. A., \& Richards, E. A. 2000, ApJ, 544, 641
Heiles, C. 1994, ApJ, 436, 720

Helou, G., Khan, I. R., Malek, L., \& Boahmer, L. 1988, ApJS, 68, 151

Hopkins, A. M., Connolly, A. J., Haarsma, D. B., \& Cram, L. E. 2001, AJ, 122, 288

Jacoby, G. H., Hunter, D. A., \& Christian, C. A. 1984, ApJS, 56, 257

Jansen, R. A., Frax, M., \& Fabricant, D. 2001, ApJ, 551, 825

Kaufman, M. J., Wolfire, M. G., Hollenbach, D. J., \& Luhman, M. L. 1999, ApJ, 527, 795

Kennicutt, R. C. 1983, ApJ, 272, 54

Kennicutt, R. C. 1984, ApJ, 287, 116

Kennicutt, R. C. 1998, ARA\&A, 36, 189

Kewley, L. J., Dopita, M. A., Sutherland, R. S., Heisler, C. A., \& Trevena, J. 2001, ApJ, 556, 121

Kurucz, R. 1993, ATLAS9 Stellar Atmosphere Programs and 2 km/s grid. Kurucz CD-ROM No. 13, Cambridge, Mass.: Smithsonian Astrophysical Observatory, 13

Lejeune, T., Cuisinier, F., \& Buser, R. 1998, A\&AS, 130, 65

Lennon, D. J., \& Burke, V. M. 1994, A\&AS, 103, 273

Malhotra, S., Kaufman, M. J., Hollenbach, D., et al. 2001, ApJ, 561, 766

McGaugh, S. S. 1991, ApJ, 380, 140

Meurer, G. R., Heckman, T. M., \& Calzetti, D. 1999, ApJ, 521, 64

Moy, E., Rocca-Volmerange, B., \& Fioc, M. 2001, A\&A, 365, 347 (MRF01)

Nussbaumer, H., \& Schmutz, W. 1984, A\&A, 138, 495

Oey, M. S., \& Shields, J. C. 2000, ApJ, 539, 687

Osterbrock, D. E. 1989, Astrophysics of Gaseous Nebulae and Active Galactic Nuclei (Mill Valley, CA: Univ. Science Books)

Peng, J. F., \& Pradhan, A. K. 1995, A\&AS, 112, 151

Perez-Olea, D. E., \& Colina, L. 1995, MNRAS, 277, 857

Pickles, A. J. 1998, PASP, 110, 863

Poggianti, B. M., Bressan, A., \& Franceschini, A. 2001, ApJ, 550, 195

Rigopoulou, D., Spoon, H. W. W., Genzel, R., et al. 1999, AJ, 118, 2625

Rodighiero, G., Granato, G. L., Franceschini, A., Fasano, G., \& Silva, L. 2000, A\&A, 364, 517

Rubin, R. H., Simpson, J. P., Lord, S. D., et al. 1994, ApJ, 420, 772

Rubin, R. H., Dufour, R. J., Geballe, T. R., et al. 2001, in Spectroscopic Challenges of Photoionized Plasmas, ed. G. Ferland, \& D. W. Savin, ASP Conf. Ser., 247, 479

Sanders, D. B., \& Mirabel, I. F. 1996, ARA\&A, 34, 749

Schaerer, D., Contini, T., \& Kunth, D. 1999, A\&A, 341, 399

Schaerer, D., de Koter, A., Schmutz, W., \& Maeder, A. 1996, A\&A, $312,475 \mathrm{~S}$

Schmutz, W., Leitherer, C., \& Gruenwald, R. 1992, PASP, 104, 1164

Silva, L., Granato, G. L., Bressan, A., \& Danese, L. 1998, ApJ, 509, 103

Soifer, B. T., Boehmer, L., Neugebaruer, G., \& Sanders, D. B. 1989, AJ, 98, 766

Soifer, B. T., Sanders, D. B., Neugebauer, G., et al. 1986, ApJ, 303, L41

Stasińska, G., Schaerer, D., \& Leitherer, C. 2001, A\&A, 370, 1

Storey, P. J., \& Hummer, D. G. 1995, MNRAS, 272, 41

Sullivan, M., Treyer, M. A., Ellis, R. S., et al. 2000, MNRAS, 312, 442

Sullivan, M., Mobasher, B., Chan, B., et al. 2001, ApJ, 558, 72

Terlevich, R., Melnick, J., Masegosa, M., Moles, M., \& Copetti, M. V. F. 1991, A\&AS, 91, 285

Veilleux, S., \& Osterbrock, D. E. 1987, ApJS, 63, 295

Verma, A., Lutz, D., Sturm, E., et al. 2003, A\&A, 403, 829 